\title{
Risk-sensitive control for a class of nonlinear systems with multiplicative noise
}

\author{
Paresh Date $^{1}$ and Bujar Gashi ${ }^{2}$
}

\begin{abstract}
In this paper, we consider the problem of optimal control for a class of nonlinear stochastic systems with multiplicative noise. The nonlinearity consists of quadratic terms in the state and control variables. The optimality criteria are of a risk-sensitive and generalised risk-sensitive type. The optimal control is found in an explicit closed-form by the completion of squares and the change of measure methods. As applications, we outline two special cases of our results. We show that a subset of the class of models which we consider leads to a generalized quadratic affine term structure model (QATSM) for interest rates. We also demonstrate how our results lead to generalisation of exponential utility as a criterion in optimal investment.
\end{abstract}

Keywords: Risk-sensitive control; Nonlinear systems; Bond pricing;

Optimal investment.

\section{Introduction}

Let $(\Omega, \mathcal{F}, \mathbb{P})$ be a complete probability space, on which an $n$-dimensional standard Brownian motion $(W(t), t \geq 0)$, is defined. We assume that $\mathcal{F}_{t}$ is the augmentation of $\sigma\{W(s) \mid 0 \leq s \leq t\}$ by all the $\mathbb{P}$-null sets of $\mathcal{F}$. Let $f:[0, T] \times \Omega \rightarrow \mathbb{R}^{n}$ denote an $\left\{\mathcal{F}_{t}\right\}_{t \geq 0}$ adapted process; if $\mathbb{E} \int_{0}^{T}|f(t)|^{2} d t<\infty$, we write $f(\cdot) \in L_{\mathcal{F}}^{2}\left(0, T ; \mathbb{R}^{n}\right)$.

\footnotetext{
${ }^{1}$ Centre for the Analysis of Risk ad Optimisation Modelling Applications (CARISMA), Department of Mathematical Sciences, Brunel University, Uxbridge, UB8 3PH, UK; Email: paresh.date@brunel.ac.uk

${ }^{2}$ Institute of Financial and Actuarial Mathematics (IFAM), Department of Mathematical Sciences, The University of Liverpool, Liverpool, L69 7ZL, UK; Email: Bujar.Gashi@liverpool.ac.uk
} 
Consider the linear stochastic control system:

$$
\left\{\begin{array}{l}
d x_{1}(t)=\left[A x_{1}(t)+B u(t)\right] d t+\sum_{j=1}^{n} C_{j} d W_{j}(t), \\
x_{1}(0)=x_{10} .
\end{array}\right.
$$

The initial state $x_{10}$ is a Gaussian random variable with mean $\mu_{0}$ and variance $P_{0}$, with $P_{0}$ being nonsingular. It is assumed that $x_{10}$ and $W(t)$ are independent objects. The rest of the given data are:

$$
\begin{aligned}
& A(\cdot) \in L^{\infty}\left(0, T ; \mathbb{R}^{n_{1} \times n_{1}}\right) ; \quad B(\cdot) \in L^{\infty}\left(0, T ; \mathbb{R}^{n_{1} \times m}\right) ; \\
& C_{j}(\cdot) \in L^{\infty}\left(0, T ; \mathbb{R}^{n_{1}}\right), \quad j=1, \ldots, n .
\end{aligned}
$$

Here $L^{\infty}(\cdot)$ denotes the set of uniformly bounded functions. For notational simplicity, we do not indicate explicitly the time dependence of coefficients. When the control process $u(\cdot) \in L_{\mathcal{F}}^{2}\left(0, T ; \mathbb{R}^{m}\right)$, equation (1.1) has a unique strong solution (see, e.g. Theorem 1.6.14 of [36]). $u(\cdot)$ is typically chosen by minimising a real-valued functional of $x_{1}(\cdot)$ and $u(\cdot)$ over $u(\cdot)$. A cost function of interest here is risk-sensitive cost functional:

$J_{1}(u(\cdot))=\gamma \mathbb{E}\left\{\exp \left[\frac{\gamma}{2} x_{1}^{\prime}(T) S x_{1}(T)+\frac{\gamma}{2} \int_{0}^{T}\left[x_{1}^{\prime}(t) Q x_{1}(t)+u^{\prime}(t) R u(t)\right] d t\right]\right\}$

where $\gamma \in \mathbb{R}, \gamma \neq 0$, is a given constant. The coefficient matrices are assumed to be symmetric and belong to the following spaces:

$$
Q(\cdot) \in L^{\infty}\left(0, T ; \mathbb{R}^{n_{1} \times n_{1}}\right), \quad R(\cdot) \in L^{\infty}\left(0, T ; \mathbb{R}^{m \times m}\right), \quad S \in \mathbb{R}^{n_{1} \times n_{1}},
$$

with $Q(t) \geq 0, R(t)>0$, a.e. $t \in[0, T]$, and $S \geq 0$.

The optimal control problem of finding $u(\cdot)$ that minimises (1.3) subject to (1.1), was introduced by Jacobson in [19]. Assuming full state observation, Jacobson has given a complete solution to this problem. The optimal control is of a linear state-feedback form, and has great similarity with the linear-quadratic control [35]. An important difference, however, is that in the risk-sensitive case the optimal control depends on the intensity of noise $C_{j}(t)$, $j=1, \ldots, n$, which is not the case for the linear-quadratic control. Jacobson 
also solves the discrete-time version of the problem and explores the relation with differential games.

After this pioneering work, several attempts were made in solving the partial observation problem by [22], [23], [31], [32]. However, it is only in [6] that the complete solution to this problem was finally obtained. In the recent paper [11], the authors have generalised all these classical results by introducing a more general risk-sensitive criterion that contains noise dependent penalties of the state and control variables. The discrete-time partial observation problem was solved by Whittle in [33] (see also [34]). For infinite horizon criterion in a Markovian setting, the reader can consult [5], [9], [10]. An important relation with robust controllers was found in [14], [15], whereas the risk-sensitive maximum principle was studied in [26], [27], [17], [20]. A more general version of linear exponential quadratic control, where $x(t)$ evolves in an infinite dimensional Hilbert space is discussed in [13]. The optimal investment problem is particulary suitable for the application of risk-sensitive control; see for example [7], [12], [24], [16].

A risk-sensitive control problem with system dynamics that contains multiplicative noise, but has a linear penalty on the state was essentially solved a few years before Jacobson. This was achieved by Merton in [29], [30], who considered the problem of optimal investment with an exponential utility. The system dynamics in this case is the self-financing portfolio

$$
d y(t)=[r(t) y(t)+b(t) u(t)] d t+\sum_{i=1}^{n} \sigma_{i}(t) u(t) d W_{i}(t),
$$

where $r(t), b(t), \sigma_{i}(t)$, are market dependent known coefficients, and $u(t)$ represents the trading strategy. The aim of the investor with exponential utility is to minimise the criterion

$$
\mathbb{E}\left[e^{-a y(T)}\right]
$$

for some known coefficient $a$. Note that the cost function is exponential affine in the state, in contrast to the exponential quadratic form in equation (1.3).

In this paper we formulate a risk-sensitive control problem which contains the previously mentioned two problems as special cases. We do so by 
first extending the systems dynamics (1.1) to include multiplicative noise as follows:

$$
\left\{\begin{aligned}
d x_{1}(t) & =\left[A x_{1}(t)+B u(t)\right] d t+\sum_{j=1}^{n} C_{j} d W_{j}(t), \\
d x_{2}(t) & =\left[A_{1} x_{1}(t)+A_{2} x_{2}(t)+D\left(x_{1}(t), u(t)\right)+B_{1} u(t)\right] d t \\
& +\sum_{j=1}^{n}\left[A_{3 j} x_{1}(t)+B_{2 j} u(t)+C_{1 j}\right] d W_{j}(t), \\
x_{1}(0) & =x_{10}, \quad x_{2}(0)=x_{20}
\end{aligned}\right.
$$

The given data are such that:

$$
\begin{array}{r}
A_{1}(\cdot), A_{31}(\cdot), \ldots, A_{3 n}(\cdot) \in L^{\infty}\left(0, T ; \mathbb{R}^{n_{2} \times n_{1}}\right), \\
A_{2}(\cdot) \in L^{\infty}\left(0, T ; \mathbb{R}^{n_{2} \times n_{2}}\right), \\
B_{1}(\cdot), B_{21}(\cdot), \ldots, B_{2 n}(\cdot) \in L^{\infty}\left(0, T ; \mathbb{R}^{n_{2} \times m}\right), \\
C_{11}(\cdot), \ldots, C_{1 n}(\cdot) \in L^{\infty}\left(0, T ; \mathbb{R}^{n_{2}}\right),
\end{array}
$$

and we further assume that $x_{20}$ is a normal random variable independent of $x_{10}$ and $W(t)$. The vector $D\left(x_{1}(t), u(t)\right)$ is defined as

$$
D\left(x_{1}(t), u(t)\right)=\left[\begin{array}{c}
x_{1}^{\prime}(t) Q_{1} x_{1}(t)+u^{\prime}(t) X_{1} x_{1}(t)+u^{\prime}(t) R_{1} u(t) \\
\vdots \\
x_{1}^{\prime}(t) Q_{n_{2}} x_{1}(t)+u^{\prime}(t) X_{n_{2}} x_{1}(t)+u^{\prime}(t) R_{n_{2}} u(t)
\end{array}\right],
$$

where

$$
\begin{gathered}
Q_{1}(\cdot), \ldots, Q_{n_{2}}(\cdot) \in L^{\infty}\left(0, T ; \mathbb{R}^{n_{1} \times n_{1}}\right), \\
X_{1}(\cdot), \ldots, X_{n_{2}}(\cdot) \in L^{\infty}\left(0, T ; \mathbb{R}^{m \times n_{1}}\right), \\
R_{1}(\cdot), \ldots, R_{n_{2}}(\cdot) \in L^{\infty}\left(0, T ; \mathbb{R}^{m \times m}\right) .
\end{gathered}
$$


We also assume that $Q_{j}, R_{j}, j=1, \ldots, n_{2}$, are symmetric. Note that equation for the state $x_{2}(t)$ is nonlinear in both the state $x_{1}(t)$ and the control $u(t)$. In addition, the state $x_{1}(t)$ and control $u(t)$ multiply the sources of noise $W_{j}(t)$, $j=1, \ldots, n$.

We extend the criterion (1.3) to include a linear penalty on the newly introduced state $x_{2}(t)$ as follows:

$$
\begin{aligned}
J(u(\cdot)) & =\gamma \mathbb{E}\left\{\operatorname { e x p } \left[\frac{\gamma}{2} x_{1}^{\prime}(T) S x_{1}(T)+\frac{\gamma}{2} \int_{0}^{T}\left[x_{1}^{\prime}(t) Q x_{1}(t)+u^{\prime}(t) R u(t)\right] d t\right.\right. \\
& +\frac{\gamma}{2} S_{1}^{\prime} x_{1}(T)+\frac{\gamma}{2} S_{2}^{\prime} x_{2}(T) \\
& \left.\left.+\frac{\gamma}{2} \int_{0}^{T}\left[L_{1}^{\prime} x_{1}(t)+L_{2}^{\prime} x_{2}(t)+L_{u}^{\prime} u(t)+u^{\prime}(t) X x_{1}(t)\right] d t\right]\right\}
\end{aligned}
$$

The new matrices and vectors that appear in this criterion are such that:

$$
\begin{aligned}
& L_{1}(\cdot) \in L^{\infty}\left(0, T ; \mathbb{R}^{n_{1}}\right), \quad L_{2}(\cdot) \in L^{\infty}\left(0, T ; \mathbb{R}^{n_{2}}\right), \quad L_{u}(\cdot) \in L^{\infty}\left(0, T ; \mathbb{R}^{m}\right), \\
& X(\cdot) \in L^{\infty}\left(0, T ; \mathbb{R}^{m \times n_{1}}\right), \quad S_{1} \in \mathbb{R}^{n_{1}}, \quad S_{2} \in \mathbb{R}^{n_{2}} .
\end{aligned}
$$

Our main concern is to solve the optimal control problem of minimising (1.5) subject to (1.4). This is achieved in Sec. 2, where the optimal control is found in an explicit closed-form, subject to solving a Riccati type differential equation. The optimal control turns out to be an affine function of the state $x_{1}(t)$. Since the considered optimal control problem is of a nonlinear nature, it is rather surprising that it admits an explicit closed-form solution. Moreover, based on our extended system dynamics, we introduce a new interest rate model and derive the price of a zero-coupon bond in a closed form. Next in Sec. 3 we consider a generalised risk-sensitive control problem of the type introduced recently by the authors in [11], where noise dependent penalties of state and control variables are included in the criterion. Even for this case we obtain the solution explicitly by the change of measure method. As an interesting application of our general results, we propose an extension of the Merton's optimal investment problem with exponential utility. 


\section{Risk-sensitive control}

\subsection{Problem formulation}

In this section, we are interested in the following optimal control problem

$$
\left\{\begin{array}{l}
\min _{u(\cdot) \in \mathcal{A}} J(u(\cdot)), \\
\text { s.t. }(1.4) \text { holds, }
\end{array}\right.
$$

where $J(u(\cdot))$ is as defined in (1.5). The set of admissible controls $\mathcal{A}$ will be defined later, after some necessary notation is introduced.

We find the solution to problem (2.1) by the completion of squares method. Let $p_{2}(t)=\left[p_{21}(t), \ldots, p_{2 n_{2}}(t)\right]^{\prime}$ denote the solution to the following linear differential equation

$$
\left\{\begin{array}{l}
\dot{p}_{2}(t)+A_{2}^{\prime} p_{2}(t)+L_{2}=0 \\
p_{2}(T)=S_{2}
\end{array}\right.
$$

We define $\bar{R}(t)$ as

$$
\bar{R}(t)=R+\sum_{j=1}^{n_{2}} p_{2 j}(t) R_{j}+\frac{\gamma}{4} \sum_{j=1}^{n} B_{2 j}^{\prime} p_{2}(t) p_{2}^{\prime}(t) B_{2 j}
$$

We further introduce a Riccati type equation

$$
\left\{\begin{array}{l}
\dot{P}(t)+A^{\prime} P(t)+P(t) A+Q+\sum_{i=1}^{n_{2}} p_{2 i} Q_{i}-\frac{1}{4} \bar{X}^{\prime} \bar{R}^{-1} \bar{X} \\
\quad+\frac{\gamma}{4} \sum_{j=1}^{n}\left[2 P(t) C_{j}+A_{3 j}^{\prime} p_{2}(t)\right]\left[2 C_{j}^{\prime} P(t)+p_{2}^{\prime}(t) A_{3 j}\right]=0, \\
\bar{X}=X+2 B^{\prime} P(t)+\sum_{i=1}^{n_{2}} p_{2 i}(t) X_{i}+\frac{\gamma}{4} \sum_{j=1}^{n} 2 B_{2 j}^{\prime} p_{2}(t)\left[2 C_{j}^{\prime} P(t)+p_{2}^{\prime}(t) A_{3 j}\right], \\
P(T)=S .
\end{array}\right.
$$

Our further results rest on the following two assumptions: 
Assumption 1. $\bar{R}(t)>0, \quad$ a.e. $\quad t \in[0, T]$.

Assumption 2. The Riccati equation (2.4) has a unique global solution in the interval $[0, T]$.

The Riccati equation (2.4) can be easily rearranged so that it has the form of the Riccati equation for the deterministic linear-quadratic regulator [4], from which the following sufficient conditions for Assumption 2 to hold are derived:

$$
\left\{\begin{array}{l}
\frac{1}{4} \beta^{\prime} \bar{R}^{-1} \beta-\gamma \sum_{j=1}^{n} C_{j} C_{j}^{\prime}>0 \\
Q+\sum_{i=1}^{n_{2}} p_{2 i}(t) Q_{i}+\frac{\gamma}{4} \sum_{j=1}^{n} A_{3 j}^{\prime} p_{2} p_{2}^{\prime} A_{3 j}-\frac{1}{4} \alpha^{\prime} \bar{R}^{-1} \alpha \geq 0 \\
\alpha \equiv X+\sum_{i=1}^{n_{2}} p_{2 i}(t) X_{i}+\frac{\gamma}{2} \sum_{j=1}^{n} B_{2 j}^{\prime} p_{2}(t) p_{2}^{\prime}(t) A_{3 j} \\
\beta \equiv 2 B^{\prime}+\gamma \sum_{j=1}^{n} B_{2 j}^{\prime} p_{2}(t) C_{j}^{\prime} .
\end{array}\right.
$$

We now introduce two linear differential equations, which have a unique solution under Assumption 2.

$$
\left\{\begin{array}{l}
\dot{p}_{1}+L_{1}+A^{\prime} p_{1}(t)+A_{1}^{\prime} p_{2}(t)-\frac{1}{2} \bar{X}^{\prime} \bar{R}^{-1} \bar{Y}+\frac{\gamma}{2} \bar{Z}=0, \\
\bar{Z}=\sum_{j=1}^{n}\left[2 P(t) C_{j}+A_{3 j}^{\prime} p_{2}(t)\right]\left[C_{j}^{\prime} p_{1}(t)+C_{1 j}^{\prime} p_{2}(t)\right], \\
\bar{Y}=L_{u}+B^{\prime} p_{1}(t)+B_{1}^{\prime} p_{2}(t)+\frac{\gamma}{4} \sum_{j=1}^{n} 2 B_{2 j}^{\prime} p_{2}(t)\left[C_{j}^{\prime} p_{1}(t)+C_{1 j}^{\prime} p_{2}(t)\right], \\
p_{1}(T)=S_{1},
\end{array}\right.
$$




$$
\left\{\begin{array}{l}
\dot{p}+\sum_{j=1}^{n} C_{j}^{\prime} P(t) C_{j}+\frac{\gamma}{4} \sum_{j=1}^{n}\left[p_{1}^{\prime}(t) C_{j}+p_{2}^{\prime}(t) C_{1 j}\right]\left[C_{j}^{\prime} p_{1}(t)+C_{1 j}^{\prime} p_{2}(t)\right]=0, \\
p(T)=0 .
\end{array}\right.
$$

In order to define the set of admissible controls $\mathcal{A}$ we introduce the processes $v(t)$ and $H(t)$ as:

$$
\begin{gathered}
\left\{\begin{array}{l}
d v(t)=\left[x_{1}^{\prime}(t) Q x_{1}(t)+u^{\prime}(t) R u(t)+u^{\prime}(t) X x_{1}(t)+L_{1}^{\prime} x_{1}(t)+L_{2}^{\prime} x_{2}(t)+L_{u}^{\prime} u(t)\right] d t \\
v(0)=0
\end{array}\right. \\
H(t) \equiv v(t)+x_{1}^{\prime}(t) P(t) x_{1}(t)+p(t)+p_{1}^{\prime}(t) x_{1}(t)+p_{2}^{\prime}(t) x_{2}(t) .
\end{gathered}
$$

We introduce the following conditions for the control process:

C1(q). $\mathbb{E}\left[\left[x_{1}^{\prime}(t)\left(2 P C_{j}+A_{3 j}^{\prime} p_{2}\right)+p_{2}^{\prime} B_{2 j} u(t)+p_{1}^{\prime} C_{j}+p_{2}^{\prime} C_{1 j}\right]^{\frac{2 q}{q-1}}\right]<\infty, j=$ $1, \ldots, n$, for some $q>1$, a.e. $t \in[0, T]$.

C2(q). $\mathbb{E}\left[e^{q \gamma H(t)}\right]<\infty, \quad$ for some $q>1$ and $\forall t \in[0, T]$.

Definition 1. Let $\mathcal{A}_{q}=\left\{u(\cdot) \in L_{\mathcal{F}}^{2}\left(0, T ; \mathbb{R}^{m}\right): C 1(q)\right.$ and $C \mathscr{2}(q)$ hold $\}$. The set of admissible controls $\mathcal{A}$ for the problem (2.1) is

$$
\mathcal{A}=\bigcup_{q>1} \mathcal{A}_{q} .
$$

Constraining the controls to be square integrable ensures that (1.4) has a unique solution. Indeed, in this case the equation for $x_{1}(t)$ has a unique solution. Since the nonlinearity appearing in the equation for $x_{2}(t)$ depends only on $x_{1}(t)$ and $u(t)$, such an equation also has a unique solution. The constraints $C 1(q)$ and $C 2(q)$ are imposed in order to be able to apply the completion of squares method, and may be stronger than necessary. However, note that constraint $C 2(q)$ implies the necessary requirement of $J(u(\cdot))<\infty$ for all $u(\cdot) \in \mathcal{A}$. Indeed, the following inequality holds

$$
J(u(\cdot))=\gamma \mathbb{E}\left[e^{\frac{\gamma}{2} H(T)}\right] \leq \gamma\left(\mathbb{E}\left[e^{q \gamma H(T)}\right]\right)^{\frac{1}{2 q}}<\infty .
$$




\subsection{Affine controls}

In this section we derive sufficient conditions that ensure the control processes affine in state $x_{1}(t)$ belong to the admissible set $\mathcal{A}$. These conditions will be used later is proving our main result (the solution to problem (2.1)). Hence, let us consider the control process given by

$$
\bar{u}(t)=K_{0}+K_{1} x_{1}(t),
$$

where $K_{0}(\cdot) \in L^{\infty}\left(0, T ; \mathbb{R}^{m}\right)$ and $K_{1}(\cdot) \in L^{\infty}\left(0, T ; \mathbb{R}^{m \times n_{1}}\right)$ are given matrices. Substituting (2.7) in the equation for $x_{1}(t)$ gives

$$
d x_{1}=\left(\bar{A} x_{1}+\bar{B}\right) d t+\sum_{j=1}^{n} C_{j} d W_{j}
$$

where $\bar{A}=A+B K_{1}, \bar{B}=B K_{0}$. Since $x_{1}(t)$ is a square integrable process, so is $\bar{u}$. The condition $\mathrm{C} 1(\mathrm{q})$ is also satisfied due to the bounded expected powers of $x_{1}(t)$ (see, e. g. Theorem 4.5.4 of [21]). It thus remains to show that condition $\mathrm{C} 2(\mathrm{q})$ holds.

Substituting (2.7) in the equation for $x_{2}(t)$ gives

$$
d x_{2}=\left[\bar{A}_{1} x_{1}+A_{2} x_{2}+\bar{D}\left(x_{1}\right)\right] d t+\sum_{j=1}^{n}\left(\bar{A}_{3 j} x_{1}+\bar{C}_{1 j}\right) d W_{j}
$$

where $\bar{A}_{1}=A_{1}+B_{1} K_{1}$, and $\bar{A}_{3 j}=A_{3 j}+B_{2 j} K_{1}, \bar{C}_{1 j}=B_{2 j} K_{0}+C_{1 j}$, $j=1, \ldots, n$. The vector $D\left(x_{1}\right)$ is defined as

$$
\bar{D}\left(x_{1}\right)=\left[\begin{array}{c}
\bar{Q}_{10}+\bar{Q}_{11} x_{1}+x_{1}^{\prime} \bar{Q}_{12} x_{1} \\
\vdots \\
\bar{Q}_{n_{2} 0}+\bar{Q}_{n_{2} 1} x_{1}+x_{1}^{\prime} \bar{Q}_{n_{2} 2} x_{1}
\end{array}\right],
$$

where for $j=1, \ldots, n_{2}$ we have

$$
\begin{aligned}
\bar{Q}_{j 0} & =K_{0}^{\prime} R_{j} K_{0} \\
\bar{Q}_{j 1} & =K_{0}^{\prime} X_{j}+2 K_{0}^{\prime} R_{j} K_{1}, \\
\bar{Q}_{j 2} & =Q_{j}+K_{1}^{\prime} X_{j}+X_{j}^{\prime} K_{1}+K_{1}^{\prime} R_{j} K_{1} .
\end{aligned}
$$


Next we find $H(t)$ under the control $\bar{u}(t)$. Note that

$$
\begin{aligned}
\int_{0}^{t} L_{2}^{\prime} x_{2} d s+p_{2}^{\prime} x_{2} & =p_{2}^{\prime}(0) x_{2}(0)+\int_{0}^{t}\left[p_{2}^{\prime} \bar{A}_{1} x_{1}+\left(\dot{p}_{2}^{\prime}+p_{2}^{\prime} A_{2}+L_{2}^{\prime}\right) x_{2}+p_{2}^{\prime} \bar{D}\left(x_{1}\right)\right] d t \\
& +\sum_{j=1}^{n} \int_{0}^{t}\left(p_{2}^{\prime} \bar{A}_{3 j} x_{1}+p_{2}^{\prime} \bar{C}_{1 j}\right) d W_{j} \\
& =p_{2}^{\prime}(0) x_{2}(0)+\int_{0}^{t}\left[p_{2}^{\prime} \bar{A}_{1} x_{1}+p_{2}^{\prime} \bar{D}\left(x_{1}\right)\right] d t \\
& +\sum_{j=1}^{n} \int_{0}^{t}\left(p_{2}^{\prime} \bar{A}_{3 j} x_{1}+p_{2}^{\prime} \bar{C}_{1 j}\right) d W_{j}
\end{aligned}
$$

The product $p_{2}^{\prime} \bar{D}\left(x_{1}\right)$ can be written as

$$
p_{2}^{\prime} \bar{D}\left(x_{1}\right)=\sum_{j=1}^{n_{2}} p_{2 j} \bar{Q}_{j 0}+x_{1}^{\prime} \sum_{j=1}^{n_{2}} p_{2 j} \bar{Q}_{j 1}^{\prime}+x_{1}^{\prime}\left(\sum_{j=1}^{n_{2}} p_{2 j} \bar{Q}_{j 2}\right) x_{1} .
$$

The function $H(t)$ under the control $\bar{u}(t)$ can now be written as

$$
\begin{aligned}
H(t) & =p_{2}^{\prime} x_{2}(0)+p(t)+\int_{0}^{t}\left[\left(\sum_{j=1}^{n_{2}} p_{2 j} \bar{Q}_{j 0}\right)+K_{0}^{\prime} R K_{0}+L_{u}^{\prime} K_{0}\right] d t \\
& +\int_{0}^{t}\left[x_{1}^{\prime}\left(Q+K_{1}^{\prime} R K_{1}+\frac{1}{2} K_{1}^{\prime} X+\frac{1}{2} X^{\prime} K_{1}+\sum_{j=1}^{n_{2}} p_{2 j} \bar{Q}_{j 2}\right) x_{1}\right. \\
& \left.+x_{1}^{\prime}\left(2 K_{1}^{\prime} R K_{0}+X^{\prime} K_{0}+L_{1}+K_{1}^{\prime} L_{u}+\bar{A}_{1}^{\prime} p_{2}+\sum_{j=1}^{n_{2}} p_{2 j} \bar{Q}_{j 1}^{\prime}\right)\right] d t \\
& +\sum_{j=1}^{n} \int_{0}^{t}\left(p_{2}^{\prime} \bar{A}_{3 j} x_{1}+p_{2}^{\prime} C_{1 j}\right) d W_{j}+x_{1}^{\prime} P(t) x_{1}+p_{1}^{\prime}(t) x_{1} .
\end{aligned}
$$

For a symmetric and differentiable function $M(t)$ and a differentiable function $N(t)$, of dimensions $n_{1} \times n_{1}$ and $n_{1} \times 1$, respectively, such that $M(0) \geq 0$ 
and $N(0)=0$, the following holds:

$$
\begin{aligned}
0 & =-x_{1}^{\prime} M(t) x_{1}-x_{1}^{\prime} N(t)+x_{1}^{\prime}(0) M(0) x_{1}(0)+\int_{0}^{t}\left[x_{1}^{\prime}\left(\dot{M}+M \bar{A}+\bar{A}^{\prime} M\right) x_{1}+x_{1}^{\prime}(2 M \bar{B})\right. \\
& \left.+\sum_{j=1}^{n}\left(C_{j}^{\prime} M C_{j}\right)+x_{1}^{\prime} \dot{N}+x_{1}^{\prime} \bar{A}^{\prime} N+N^{\prime} \bar{B}\right] d t \\
& +\sum_{j=1}^{n} \int_{0}^{t}\left(x_{2}^{\prime} 2 M C_{j}+N^{\prime} C_{j}\right) d W_{j} .
\end{aligned}
$$

Adding this to the right-hand side of $H(t)$ gives:

$$
\begin{aligned}
H(t) & =p_{2}^{\prime} x_{2}(0)+p(t)+x_{1}^{\prime}(0) M(0) x_{1}(0)+\int_{0}^{t}\left[\left(\sum_{j=1}^{n_{2}} p_{2 j} \bar{Q}_{j 0}\right)+K_{0}^{\prime} R K_{0}+L_{u}^{\prime} K_{0}\right] d t \\
& +\int_{0}^{t}\left[\sum_{j=1}^{n}\left(C_{j}^{\prime} M C_{j}\right)+N^{\prime} \bar{B}\right] d t+x_{1}^{\prime}[P(t)-M(t)] x_{1}+x_{1}^{\prime}\left[p_{1}^{\prime}(t)-N(t)\right] \\
& +\int_{0}^{t}\left[x_{1}^{\prime}\left(Q+K_{1}^{\prime} R K_{1}+\frac{1}{2} K_{1}^{\prime} X+\frac{1}{2} X^{\prime} K_{1}+\sum_{j=1}^{n_{2}} p_{2 j} \bar{Q}_{j 2}+\dot{M}+M \bar{A}+\bar{A}^{\prime} M\right) x_{1}\right. \\
& +x_{1}^{\prime}\left(2 K_{1}^{\prime} R K_{0}+X^{\prime} K_{0}+L_{1}+K_{1}^{\prime} L_{u}+\bar{A}_{1}^{\prime} p_{2}+\sum_{j=1}^{n_{2}} p_{2 j} \bar{Q}_{j 1}^{\prime}\right. \\
& \left.\left.+2 M \bar{B}+\dot{N}+\bar{A}^{\prime} N\right)\right] d t \\
& +\sum_{j=1}^{n} \int_{0}^{t}\left[x_{1}^{\prime}\left(\bar{A}_{3 j}^{\prime} p_{2}+2 M C_{j}\right)+p_{2}^{\prime} C_{1 j}+N^{\prime} C_{j}\right] d W_{j} .
\end{aligned}
$$

Define the matrices $M_{1}(t)$ and $N_{1}(t)$ as:

$$
\begin{aligned}
M_{1} & \equiv\left[\bar{A}_{31}^{\prime} p_{2}+2 M C_{1}, \ldots, \bar{A}_{3 n}^{\prime} p_{2}+2 M C_{n}\right], \\
& =\bar{A}_{3} p_{2}+2 M \bar{C}, \\
N_{1} & \equiv\left[p_{2}^{\prime} C_{11}+N^{\prime} C_{1}, \ldots, p_{2}^{\prime} C_{1 n}+N^{\prime} C_{n}\right] .
\end{aligned}
$$

where $\bar{A}=\left[\bar{A}_{31}^{\prime}, \ldots, \bar{A}_{3 n}^{\prime}\right]$, and $\bar{C}=\left[C_{1}, \ldots, C_{n}\right]$. The stochastic integrals of (2.11) multiplied by the constant $\gamma q$ can be written in the following more 
convenient form

$$
\begin{aligned}
& \gamma q \sum_{j=1}^{n} \int_{0}^{t}\left[x_{1}^{\prime}\left(\bar{A}_{3 j}^{\prime} p_{2}+2 M C_{j}\right)+p_{2}^{\prime} C_{1 j}+N^{\prime} C_{j}\right] d W_{j} \\
= & \int_{0}^{t} \gamma q\left(x_{1}^{\prime} M_{1}+N_{1}\right) d W=\frac{1}{2} \int_{0}^{t} \gamma^{2} q^{2} q_{1}\left(x_{1}^{\prime} M_{1}+N_{1}\right)\left(M_{1}^{\prime} x_{1}+N_{1}^{\prime}\right) d t \\
- & \frac{1}{q_{1}} \frac{1}{2} \int_{0}^{t} q_{1}^{2} \gamma^{2} q^{2}\left(x_{1}^{\prime} M_{1}+N_{1}\right)\left(M_{1}^{\prime} x_{1}+N_{1}^{\prime}\right) d t+\frac{1}{q_{1}} \int_{0}^{t} q_{1} \gamma q\left(x_{1}^{\prime} M_{1}+N_{1}\right) d W
\end{aligned}
$$

where $1<q_{1} \in \mathbb{R}$. The function $\gamma q H(t)$ can now be written as:

$$
\begin{aligned}
& \gamma q H(t)=\gamma q\left[p_{2}^{\prime} x_{2}(0)+p(t)+\int_{0}^{t}\left[\left(\sum_{j=1}^{n_{2}} p_{2 j} \bar{Q}_{j 0}\right)+K_{0}^{\prime} R K_{0}+L_{u}^{\prime} K_{0}+\frac{1}{2} N_{1} N_{1}^{\prime} \gamma q q_{1}\right] d t\right. \\
& \left.+x_{1}^{\prime}(0) M(0) x_{1}(0)+\int_{0}^{t}\left[\sum_{j=1}^{n}\left(C_{j}^{\prime} M C_{j}\right)+N^{\prime} \bar{B}\right] d t+x_{1}^{\prime}[P(t)-M(t)] x_{1}+x_{1}^{\prime}\left[p_{1}^{\prime}(t)-N(t)\right]\right] \\
& +\gamma q \int_{0}^{t}\left[x _ { 1 } ^ { \prime } \left(Q+K_{1}^{\prime} R K_{1}+\frac{1}{2} K_{1}^{\prime} X+\frac{1}{2} X^{\prime} K_{1}+\sum_{j=1}^{n_{2}} p_{2 j} \bar{Q}_{j 2}+\right.\right. \\
& \left.\dot{M}+M \bar{A}+\bar{A}^{\prime} M+\frac{1}{2} q_{1} \gamma q M_{1} M_{1}^{\prime}\right) x_{1} \\
& +x_{1}^{\prime}\left(2 K_{1}^{\prime} R K_{0}+X^{\prime} K_{0}+L_{1}+K_{1}^{\prime} L_{u}\right. \\
& \left.\left.+\bar{A}_{1}^{\prime} p_{2}+\sum_{j=1}^{n_{2}} p_{2 j} \bar{Q}_{j 1}^{\prime}+2 M \bar{B}+\bar{N}^{\prime}+\bar{A}^{\prime} N+\gamma q q_{1} M_{1} N_{1}^{\prime}\right)\right] d t \\
& \quad \frac{1}{q_{1}} \frac{1}{2} \int_{0}^{t} q_{1}^{2} \gamma^{2} q^{2}\left(x_{1}^{\prime} M_{1}+N_{1}\right)\left(M_{1}^{\prime} x_{1}+N_{1}^{\prime}\right) d t+\frac{1}{q_{1}} \int_{0}^{t} q_{1} \gamma q\left(x_{1}^{\prime} M_{1}+N_{1}\right) d W .
\end{aligned}
$$

We choose the functions $M(t)$ and $N(t)$ to be solutions to the following Riccati and linear differential equations, respectively:

$$
\left\{\begin{array}{l}
\dot{M}+M \bar{A}+\bar{A}^{\prime} M+Q+K_{1}^{\prime} R K_{1}+\frac{1}{2} K_{1}^{\prime} X+\frac{1}{2} X^{\prime} K_{1}+\sum_{j=1}^{n_{2}} p_{2 j} \bar{Q}_{j 2}+\frac{1}{2} q_{1} \gamma q M_{1} M_{1}^{\prime}=0, \\
M(0) \geq 0,
\end{array}\right.
$$




$$
\left\{\begin{array}{l}
\dot{N}+\bar{A}^{\prime} N+\gamma q q_{1} M_{1} N_{1}^{\prime}+2 K_{1}^{\prime} R K_{0}+X^{\prime} K_{0}+L_{1}+K_{1}^{\prime} L_{u}+\bar{A}_{1}^{\prime} p_{2} \\
+\sum_{j=1}^{n_{2}} p_{2 j} \bar{Q}_{j 1}^{\prime}+2 M \bar{B}=0 \\
N(0)=0 .
\end{array}\right.
$$

Assumption 3. There exists a unique solution to (2.12).

A simple sufficient condition for this assumption to hold is (see, e.g. [4]):

$$
Q+K_{1}^{\prime} R K_{1}+K_{1}^{\prime} X+X^{\prime} K_{1}+\sum_{j=1}^{n_{2}} p_{2 j} \bar{Q}_{j 2}+q_{1} \gamma q \bar{A}_{3} p_{2} p_{2}^{\prime} \bar{A}_{3}^{\prime} \geq 0
$$

if $\gamma<0$, and with a " $\leq$ " sign if $\gamma>0$ (due to the time change $t^{\prime}=T-t$ ).

The process $\gamma q H(t)$ now becomes:

$$
\begin{aligned}
& \gamma q H(t)=\gamma q\left[p_{2}^{\prime} x_{2}(0)+p(t)+\int_{0}^{t}\left[\left(\sum_{j=1}^{n_{2}} p_{2 j} \bar{Q}_{j 0}\right)+K_{0}^{\prime} R K_{0}+L_{u}^{\prime} K_{0}+\frac{1}{2} N_{1} N_{1}^{\prime} \gamma q q_{1}\right] d t\right. \\
& \left.+x_{1}^{\prime}(0) M(0) x_{1}(0)+\int_{0}^{t}\left[\sum_{j=1}^{n}\left(C_{j}^{\prime} M C_{j}\right)+N^{\prime} \bar{B}\right] d t+x_{1}^{\prime}[P(t)-M(t)] x_{1}+x_{1}^{\prime}\left[p_{1}^{\prime}(t)-N(t)\right]\right] \\
& -\frac{1}{q_{1}} \frac{1}{2} \int_{0}^{t} q_{1}^{2} \gamma^{2} q^{2}\left(x_{1}^{\prime} M_{1}+N_{1}\right)\left(M_{1}^{\prime} x_{1}+N_{1}^{\prime}\right) d t+\frac{1}{q_{1}} \int_{0}^{t} q_{1} \gamma q\left(x_{1}^{\prime} M_{1}+N_{1}\right) d W
\end{aligned}
$$

The expected value for the exponential of $\gamma q H(t)$ after applying Hölder's inequality becomes:

$$
\begin{aligned}
& \mathbb{E}\left[e^{\gamma q H(t)}\right] \\
& \leq e^{\gamma q\left[p(t)+\int_{0}^{t}\left(\sum_{j=1}^{n_{2}} p_{2 j} \bar{Q}_{j 0}+K_{0}^{\prime} R K_{0}+L_{u}^{\prime} K_{0}+\frac{1}{2} N_{1} N_{1}^{\prime} \gamma q q_{1}+\sum_{j=1}^{n}\left(C_{j}^{\prime} M C_{j}\right)+N^{\prime} \bar{B}\right) d t\right]} \\
& \times \mathbb{E}\left[e^{\gamma q p_{2}^{\prime} x_{2}(0)}\right] \mathbb{E}\left[e^{\gamma q x_{1}^{\prime}(0) M(0) x_{1}(0)}\right]\left(\mathbb{E}\left[e^{x_{1}^{\prime}[P(t)-M(t)] x_{1}+x_{1}^{\prime}\left[p_{1}^{\prime}(t)-N(t)\right]}\right]^{\frac{\gamma q q_{1}}{q_{1}-1}}\right)^{\frac{q_{1}-1}{q_{1}}} \\
& \times\left(\mathbb{E}\left[e^{-\frac{1}{2} \int_{0}^{t} q_{1}^{2} \gamma^{2} q^{2}\left(x_{1}^{\prime} M_{1}+N_{1}\right)\left(M_{1}^{\prime} x_{1}+N_{1}^{\prime}\right) d t+\int_{0}^{t} q_{1} \gamma q\left(x_{1}^{\prime} M_{1}+N_{1}\right) d W}\right]\right)^{\frac{1}{q_{1}}} \\
& \leq c(t)\left(\mathbb { E } \left[e^{\left.\left.x_{1}^{\prime}[P(t)-M(t)] x_{1}+x_{1}^{\prime}\left[p_{1}^{\prime}(t)-N(t)\right]\right]^{\frac{\gamma q q_{1}}{q_{1}-1}}\right)^{\frac{q_{1}-1}{q_{1}}}},\right.\right.
\end{aligned}
$$


where

$$
\begin{aligned}
c(t)= & e^{\gamma q\left[p(t)+\int_{0}^{t}\left(\sum_{j=1}^{n_{2}} p_{2 j} \bar{Q}_{j 0}+K_{0}^{\prime} R K_{0}+L_{u}^{\prime} K_{0}+\frac{1}{2} N_{1} N_{1}^{\prime} \gamma q q_{1}+\sum_{j=1}^{n}\left(C_{j}^{\prime} M C_{j}\right)+N^{\prime} \bar{B}\right) d t\right]} \\
& \times \mathbb{E}\left[e^{\gamma q p_{2}^{\prime} x_{2}(0)}\right] \mathbb{E}\left[e^{\gamma q x_{1}^{\prime}(0) M(0) x_{1}(0)}\right]<\infty, \quad \forall t \in[0, T] .
\end{aligned}
$$

It remains to derive conditions under which

$$
\mathbb{E}\left[e^{\frac{\gamma q q_{1}}{q_{1}-1} x_{1}^{\prime}[P(t)-M(t)] x_{1}+\frac{\gamma q q_{1}}{q_{1}-1} x_{1}^{\prime}\left[p_{1}^{\prime}(t)-N(t)\right]}\right]=\mathbb{E}\left[e^{x_{1}^{\prime} \bar{P}_{2}(t) x_{2}+x_{1}^{\prime} \bar{P}_{1}(t)}\right]<\infty,
$$

where

$$
\bar{P}_{2}(t)=\frac{\gamma q q_{1}}{q_{1}-1}[P(t)-M(t)], \quad \bar{P}_{1}(t)=\frac{\gamma q q_{1}}{q_{1}-1}\left[p_{1}^{\prime}(t)-N(t)\right] .
$$

From equation (2.8) it follows that

$$
x_{1}(t) \sim N(\eta(t), \Sigma(t)) .
$$

Here $\eta(t)$ is the solution to the linear differential equation

$$
\left\{\begin{array}{l}
\dot{\eta}-\bar{A} \eta-\bar{B}=0, \\
\eta(0)=\mu_{0},
\end{array}\right.
$$

whereas $\Sigma(t)=\bar{P}(t)-\eta(t) \eta(t)^{\prime}$ with $\bar{P}(t)$ being the solution to

$$
\left\{\begin{array}{l}
\dot{\bar{P}}-\bar{A} \bar{P}-\bar{P} \bar{A}^{\prime}-\bar{B} \eta^{\prime}-\eta \bar{B}^{\prime}-\sum_{j=1}^{n} C_{j} C_{j}^{\prime}=0, \\
\bar{P}(0)=\mathbb{E}\left[x_{1}(0) x_{1}^{\prime}(0)\right],
\end{array}\right.
$$

Assumption 4. $\Sigma(t)>0, \forall t \in[0, T]$.

Assumption 5. $\Sigma_{1}(t) \equiv\left(\Sigma^{-1}-2 \bar{P}_{2}\right)^{-1}>0, \forall t \in[0, T]$.

Lemma 1. Let the Assumption 4 and Assumption 5 hold. Then the control process (2.7) belongs to $\mathcal{A}$. 
Proof. We only need to show that under assumptions A4 and A5 the relation (2.13) holds. Under assumption A4 the distribution $N(\eta(t), \Sigma(t))$ has a density. Therefore,

$$
\begin{aligned}
& \mathbb{E}\left[e^{x_{1}^{\prime} \bar{P}_{2}(t) x_{2}+x_{1}^{\prime} \bar{P}_{1}(t)}\right]=\int_{\mathbb{R}^{n_{1}}} e^{x^{\prime} \bar{P}_{2}(t) x+x^{\prime} \bar{P}_{1}(t)} \frac{1}{(2 \pi)^{n_{1} / 2}|\Sigma|^{1 / 2}} e^{-\frac{1}{2}(x-\eta)^{\prime} \Sigma^{-1}(x-\eta)} d x \\
& =\frac{\left|\Sigma_{1}\right|^{1 / 2}}{|\Sigma|^{1 / 2}} e^{-\frac{1}{2}\left(\bar{P}_{1}(t)+\Sigma^{-1} \eta\right)^{\prime}\left(\Sigma^{-1}-2 \bar{P}_{2}(t)\right)^{-1}\left(\bar{P}_{1}(t)+\Sigma^{-1} \eta\right)-\frac{1}{2} \eta^{\prime} \Sigma^{-1} \eta} \\
& \times \int_{\mathbb{R}^{n_{1}}} \frac{1}{(2 \pi)^{n_{1} / 2}\left|\Sigma_{1}\right|^{1 / 2}} e^{-\frac{1}{2}\left(x-\Sigma_{1}\left(\bar{P}_{1}(t)+\Sigma^{-1} \eta\right)\right)^{\prime} \Sigma_{1}^{-1}\left(x-\Sigma_{1}\left(\bar{P}_{1}(t)+\Sigma^{-1} \eta\right)\right)} d x \\
& =\frac{\left|\Sigma_{1}\right|^{1 / 2}}{|\Sigma|^{1 / 2}} e^{-\frac{1}{2}\left(\bar{P}_{1}(t)+\Sigma^{-1} \eta\right)^{\prime}\left(\Sigma^{-1}-2 \bar{P}_{2}(t)\right)^{-1}\left(\bar{P}_{1}(t)+\Sigma^{-1} \eta\right)-\frac{1}{2} \eta^{\prime} \Sigma^{-1} \eta}<\infty .
\end{aligned}
$$

Remark 1. Note that a sufficient condition for Assumption 5 to hold is $P(t)>M(t), \forall t \in[0, T]$, which, while somewhat easier to interpret, is more conservative than necessary.

\subsection{Problem solution}

We can now state and prove the main result of this section.

Theorem 1. Let the Assumption 1 and Assumption 2 hold. Let the coefficients $K_{0}(t)$ and $K_{1}(t)$ be chosen as

$$
K_{0}=-\frac{1}{2} \bar{R}^{-1} \bar{Y}, \quad K_{1}=-\frac{1}{2} \bar{R}^{-1} \bar{X} .
$$

Let the Assumption 3, Assumption 4, and Assumption 5 hold in this case. Then there exists a unique solution to problem (2.1) given by

$$
u^{*}(t)=K_{0}+K_{1} x_{1}(t) .
$$

The optimal cost functional is

$$
J^{*}=\gamma \mathbb{E}\left[\exp \left\{(\gamma / 2)\left[x_{10}^{\prime} P(0) x_{10}+p(0)+p_{1}^{\prime}(0) x_{10}+p_{2}^{\prime}(0) x_{20}\right]\right\}\right],
$$

with $P, p, p_{1}$ and $p_{2}$ being solutions of differential equations (2.4), (2.6), (2.5) and (2.2) respectively. 
Proof. The differential of $H(t)$ is:

$$
\begin{aligned}
& d H(t)=\left[x_{1}^{\prime}(t) Q x_{1}(t)+u^{\prime}(t) R u(t)+u^{\prime}(t) X x_{1}(t)+L_{1}^{\prime} x_{1}(t)+L_{2}^{\prime} x_{2}(t)+L_{u}^{\prime} u(t)\right] d t \\
& +\quad\left[x_{1}^{\prime}(t) \dot{P}(t) x_{1}(t)+x_{1}^{\prime}(t)\left(A^{\prime} P(t)+P(t) A\right) x_{1}(t)+2 u^{\prime}(t) B^{\prime} P(t) x_{1}(t)+\sum_{j=1}^{n} C_{j}^{\prime} P(t) C_{j}\right. \\
& +\quad \dot{p}(t)+\dot{p}_{1}^{\prime}(t) x_{1}(t)+p_{1}^{\prime}(t)\left(A x_{1}(t)+B u(t)\right)+\dot{p}_{2}^{\prime}(t) x_{2}(t) \\
& +\quad p_{2}^{\prime}(t)\left(A_{1} x_{1}(t)+A_{2} x_{2}(t)+D\left(x_{1}(t), u(t)\right)+B_{1} u(t)\right] d t \\
& +\quad \sum_{j=1}^{n}\left[x_{1}^{\prime}(t)\left(2 P(t) C_{j}+A_{3 j}^{\prime} p_{2}(t)\right)+p_{2}^{\prime}(t) B_{2 j} u(t)+p_{1}^{\prime}(t) C_{j}+p_{2}^{\prime}(t) C_{1 j}\right] d W_{j}(t) .
\end{aligned}
$$

Define $G(t)$ as:

$$
G(t) \equiv e^{\frac{\gamma}{2} H(t)}
$$

From the definitions of the differential equations and their initial conditions, it is clear that

$$
J(u(\cdot))=\gamma \mathbb{E}[G(T)]
$$


The differential of $G(t)$ is:

$$
\begin{aligned}
& d G(t) \quad=\frac{\gamma}{2} G(t)\left[x_{1}^{\prime}(t) Q x_{1}(t)+u^{\prime}(t) X x_{1}(t)+u^{\prime}(t) R u(t)+L_{1}^{\prime} x_{1}(t)+L_{2}^{\prime} x_{2}(t)+L_{u}^{\prime} u(t)\right. \\
& +\quad x_{1}^{\prime}(t) \dot{P}(t) x_{1}(t)+x_{1}^{\prime}(t)\left(A^{\prime} P(t)+P(t) A\right) x_{1}(t)+2 u^{\prime}(t) B^{\prime} P(t) x_{1}(t) \\
& +\quad \sum_{j=1}^{n} C_{j}^{\prime} P(t) C_{j}+\dot{p}(t)+\dot{p}_{1}^{\prime} x_{1}(t)+p_{1}^{\prime}(t)\left(A x_{1}(t)+B u(t)\right)+\dot{p}_{2}^{\prime} x_{2}(t) \\
& \left.+\quad p_{2}^{\prime}(t)\left(A_{1} x_{1}(t)+A_{2} x_{2}(t)+D\left(x_{1}(t), u(t)\right)+B_{1} u(t)\right)\right] d t \\
& +\quad \frac{G(t)}{2}\left(\frac{\gamma}{2}\right)^{2} \sum_{j=1}^{n}\left[x_{1}^{\prime}(t)\left(2 P(t) C_{j}+A_{3 j}^{\prime} p_{2}(t)\right)\left(2 C_{j}^{\prime} P(t)+p_{2}^{\prime}(t) A_{3 j}\right) x_{1}(t)\right. \\
& +\quad 2 u^{\prime}(t) B_{2 j}^{\prime} p_{2}(t)\left(2 C_{j}^{\prime} P(t)+p_{2}^{\prime} A_{3 j}\right) x_{1}(t) \\
& +\quad 2 x_{1}^{\prime}(t)\left(2 P(t) C_{j}+A_{3 j}^{\prime} p_{2}(t)\right)\left(C_{j}^{\prime} p_{1}(t)+C_{1 j}^{\prime} p_{2}(t)\right) \\
& +\quad u^{\prime}(t) B_{2 j}^{\prime} p_{2}(t) p_{2}^{\prime}(t) B_{2 j} u(t)+2 u^{\prime}(t) B_{2 j}^{\prime} p_{2}(t)\left(C_{j}^{\prime} p_{1}(t)+C_{1 j}^{\prime} p_{2}(t)\right) \\
& +\quad \sum_{j=1}^{\prime} \frac{\gamma}{2} G(t)\left[x_{1}^{\prime}(t)\left(2 P C_{j}+A_{3 j}^{\prime} p_{2}\right)+p_{2}^{\prime} B_{2 j} u(t)+p_{1}^{\prime} C_{j}+p_{2}^{\prime} C_{1 j}\right] d W_{j}(t) \\
& \left.\left.+\quad p_{2}^{\prime}(t) C_{1 j}\right)\left(C_{j}^{\prime} p_{1}(t)+C_{1 j}^{\prime} p_{2}(t)\right)\right] d t \\
& +\quad
\end{aligned}
$$


The sum of the terms that contain the control $u(t)$ and appear in the $d t$ part of $d G(t)$ is:

$$
\begin{aligned}
& u^{\prime}(t) X x_{1}(t)+u^{\prime}(t) R u(t)+L_{u}^{\prime} u(t)+2 u^{\prime}(t) B^{\prime} P(t) x_{1}(t)+p_{1}^{\prime}(t) B u(t) \\
+ & \sum_{i=1}^{n_{2}} p_{2 i}(t)\left[u^{\prime}(t) X_{i} x_{1}(t)+u^{\prime}(t) R_{i} u(t)\right]+p_{2}^{\prime}(t) B_{1} u(t) \\
+ & \frac{\gamma}{4} \sum_{j=1}^{n}\left[2 u^{\prime}(t) B_{2 j}^{\prime} p_{2}(t)\left(2 C_{j}^{\prime} P(t)+p_{2}^{\prime}(t) A_{3 j}\right) x_{1}(t)+u^{\prime}(t) B_{2 j}^{\prime} p_{2}(t) p_{2}^{\prime}(t) B_{2 j} u(t)\right. \\
+ & \left.2 u^{\prime}(t) B_{2 j}^{\prime} p_{2}(t)\left(C_{j}^{\prime} p_{1}(t)+C_{1 j}^{\prime} p_{2}(t)\right)\right] \\
= & u^{\prime}(t)\left[R+\sum_{j=1}^{n_{2}} p_{2 j}(t) R_{j}+\frac{\gamma}{4} \sum_{j=1}^{n} B_{2 j}^{\prime} p_{2}(t) p_{2}^{\prime}(t) B_{2 j}\right] u(t) \\
+ & u^{\prime}(t)\left\{X+2 B^{\prime} P(t)+\sum_{i=1}^{n_{2}} p_{2 i}(t) X_{i}+\frac{\gamma}{4} \sum_{j=1}^{n} 2 B_{2 j}^{\prime} p_{2}(t)\left[2 C_{j}^{\prime} P(t)+p_{2}^{\prime}(t) A_{3 j}\right]\right\} x_{1}(t) \\
+ & u^{\prime}(t)\left\{L_{u}+B^{\prime} p_{1}(t)+B_{1}^{\prime} p_{2}(t)+\frac{\gamma}{4} \sum_{j=1}^{n} 2 B_{2 j}^{\prime} p_{2}(t)\left[C_{j}^{\prime} p_{1}(t)+C_{1 j}^{\prime} p_{2}(t)\right]\right\} \\
= & u^{\prime}(t) \bar{R} u(t)+u^{\prime}(t) \bar{X} x_{1}(t)+u^{\prime}(t) \bar{Y}=u^{\prime}(t) \bar{R} u(t)+u^{\prime}(t)\left[\bar{X} x_{1}(t)+\bar{Y}\right]= \\
= & \frac{1}{4}\left[\bar{X} x_{1}(t)+\bar{Y}\right]^{\prime} \bar{R}^{-1}\left[\bar{X} x_{1}(t)+\bar{Y}\right], \\
& {\left[\frac{1}{2} \bar{R}^{-1}\left(\bar{X} x_{1}(t)+\bar{Y}\right)\right]^{\prime} \bar{R}\left[u(t)+\frac{1}{2} \bar{R}^{-1}\left(\bar{X} x_{1}(t)+\bar{Y}\right)\right] }
\end{aligned}
$$

where the last step is achieved via completion of squares. The sum of the terms that are quadratic in $x_{1}(t)$ that appear in the $d t$ part of $d G(t)$ is zero. 
Indeed,

$$
\begin{aligned}
& x_{1}^{\prime}(t) Q x_{1}(t)+x_{1}^{\prime}(t)\left(A^{\prime} P(t)+P(t) A\right) x_{1}(t)+x_{1}^{\prime}(t) \dot{P} x_{1}(t)+\sum_{i=1}^{n_{2}} p_{2 i}(t) x_{1}^{\prime}(t) Q_{i} x_{1}(t) \\
+ & x_{1}^{\prime}(t)\left\{\frac{\gamma}{4} \sum_{j=1}^{n}\left[2 P(t) C_{j}+A_{3 j}^{\prime} p_{2}(t)\right]\left[2 C_{j}^{\prime} P(t)+p_{2}^{\prime}(t) A_{3 j}\right]\right\} x_{1}(t) \\
- & \frac{1}{4} x_{1}^{\prime}(t) \bar{X}^{\prime} \bar{R}^{-1} \bar{X} x_{1}(t)=0
\end{aligned}
$$

due to Assumption 2. Similarly, due to our assumption on $p_{1}(t)$, the sum of the terms linear in $x_{1}(t)$ that appear in the $d t$ part of $d G(t)$ is also zero:

$$
\begin{gathered}
L_{1}^{\prime} x_{1}(t)+\dot{p}_{1}^{\prime} x_{1}(t)+p_{1}^{\prime}(t) A x_{1}(t)+p_{2}^{\prime}(t) A_{1} x_{1}(t)-\frac{1}{2} x_{1}^{\prime}(t) \bar{X}^{\prime} \bar{R}^{-1} \bar{Y} \\
+\quad x_{1}^{\prime}(t) \frac{\gamma}{4} \sum_{j=1}^{n} 2\left[2 P(t) C_{j}+A_{3 j}^{\prime} p_{2}(t)\right]\left[C_{j}^{\prime} p_{1}(t)+C_{1 j}^{\prime} p_{2}(t)\right]=0 .
\end{gathered}
$$

The sum of the terms that are linear in $x_{2}(t)$ is also zero due to our assumption on $p_{2}(t)$ :

$$
L_{2}^{\prime} x_{2}(t)+\dot{p}_{2}^{\prime}(t) x_{2}(t)+p_{2}^{\prime}(t) A_{2} x_{2}(t)=0 .
$$

The sum of the remaining terms, which are independent of the states and control, is also zero due to our assumption of $p(t)$.

We now focus on the expected value of the integral form of the $d W_{j}$ terms of $d G(t)$. Note that this expectation is

$\mathbb{E}\left\{\sum_{j=1}^{n} \frac{\gamma}{2} \int_{0}^{T} G(s)\left[x_{1}^{\prime}(s)\left(2 P C_{j}+A_{3 j}^{\prime} p_{2}\right)+p_{2}^{\prime} B_{2 j} u(s)+p_{1}^{\prime} C_{j}+p_{2}^{\prime} C_{1 j}\right] d W_{j}(s)\right\}$

If the integrands of the stochastic integrals are square integrable processes, then this expectation is zero. On the other hand, this is the case for all $u(\cdot) \in \mathcal{A}$. Indeed, for all $j=1, \ldots, n$, we have

$$
\begin{aligned}
& \int_{0}^{T} \mathbb{E}\left[G^{2}(s)\left[x_{1}^{\prime}(s)\left(2 P C_{j}+A_{3 j}^{\prime} p_{2}\right)+p_{2}^{\prime} B_{2 j} u(s)+p_{1}^{\prime} C_{j}+p_{2}^{\prime} C_{1 j}\right]^{2}\right] d t \\
\leq & \int_{0}^{T}\left(\mathbb{E}\left[G^{2 q}(s)\right]\right)^{\frac{1}{q}}\left(\mathbb{E}\left[\left[x_{1}^{\prime}(s)\left(2 P C_{j}+A_{3 j}^{\prime} p_{2}\right)+p_{2}^{\prime} B_{2 j} u(s)+p_{1}^{\prime} C_{j}+p_{2}^{\prime} C_{1 j}\right]^{\frac{2 q}{q-1}}\right]\right)^{\frac{q-1}{q}} d t .
\end{aligned}
$$


This is clearly finite for all our admissible controls. Therefore, the cost functional $J(u(\cdot))$ for all $u(\cdot) \in \mathcal{A}$ can be written as

$$
\begin{aligned}
J(u(\cdot) & =\gamma \mathbb{E}[G(0)] \\
& +\frac{\gamma^{2}}{2} \mathbb{E} \int_{0}^{T} G(t)\left[u(t)+\frac{1}{2} \bar{R}^{-1}\left(\bar{X} x_{1}(t)+\bar{Y}\right)\right]^{\prime} \bar{R}\left[u(t)+\frac{1}{2} \bar{R}^{-1}\left(\bar{X} x_{1}(t)+\bar{Y}\right)\right] \\
& \geq \gamma \mathbb{E}[G(0)] .
\end{aligned}
$$

This lower bound is achieved if and only if

$$
u(t)=u^{*}(t)=-\frac{1}{2} \bar{R}^{-1}\left(\bar{X} x_{1}(t)+\bar{Y}\right) .
$$

Assumption 3, Assumption 4, and Assumption 5 ensure that $u^{*}(\cdot) \in \mathcal{A}$.

Remark 2. If the process $x_{1}(t)$ is deterministic under the control $u^{*}(t)$, then it is not necessary to have Assumptions 3-5 for the conclusions of Theorem 1 to hold. Indeed, it is clear from section 2.2 that in this case $u^{*}(t)$ is admissible.

In summary, we have formulated a risk sensitive control problem for a specific class of nonlinear stochastic differential models and given the solution in closed-form. In section 3, we generalise this problem further and allow for a noise dependent penalty in the cost functional. First we see an application of a special case of the proposed model in the next subsection.

\subsection{Application to interest rate modelling and bond pricing}

As an important application of the results obtained thus far, in this subsection we propose a new model for the interest rate and obtain the price of a zero-coupon bond in an explicit closed-form. Hence, consider a bond market where the basic traded securities are zero-coupon bonds of different maturities [8]. One approach to modelling such a market is the so-called martingale modelling, where it is assumed that there exists a unique risk-neutral probability measure (under which all discounted assets are martingales), and the interest rate $r(t)$ is modelled directly under such a measure. Popular models of the interest rate are the affine term-structure models [8], and quadraticaffine term-structure models (QATSM) (see [2] and [25], among others). 
Let $\mathbb{P}$ denote the risk-neutral probability measure in the bond market, and consider the uncontrolled system dynamics (1.4), i.e.

$$
\left\{\begin{aligned}
d x_{1}(t) & =A x_{1}(t) d t+\sum_{j=1}^{n} C_{j} d W_{j}(t) \\
d x_{2}(t) & =\left[A_{1} x_{1}(t)+A_{2} x_{2}(t)+D\left(x_{1}(t)\right)\right] d t \\
& +\sum_{j=1}^{n}\left[A_{3 j} x_{1}(t)+C_{1 j}\right] d W_{j}(t) \\
x_{1}(0) & =x_{10}, \quad x_{2}(0)=x_{20}, \quad \text { where } x_{10}, x_{20} \text { are constant vectors }
\end{aligned}\right.
$$

where we have assumed that the coefficients $B, B_{1}, X_{i}, R_{i}, B_{2 i}$, in equation (1.4) are all zero. We propose the following model for the interest rate:

$$
r(t) \equiv x_{1}^{\prime}(t) \bar{Q} x_{1}(t)+\bar{L}_{1}^{\prime} x_{1}(t)+\bar{L}_{2}^{\prime} x_{2}(t),
$$

where the coefficients are assumed as

$\bar{Q}_{1}(\cdot) \in L^{\infty}\left(0, T ; \mathbb{R}^{n_{1} \times n_{1}}\right), \quad \bar{L}_{1}(\cdot) \in L^{\infty}\left(0, T ; \mathbb{R}^{n_{1}}\right), \quad \bar{L}_{2}(\cdot) \in L^{\infty}\left(0, T ; \mathbb{R}^{n_{2}}\right)$,

and $\bar{Q}_{1}$ is also symmetric. When $\bar{L}_{2}=0$ and/or $D\left(x_{1}(t)\right)=0$, this is identical to the traditional QATSM; see, e.g. [2] and the references therein. However, when these conditions are not satisfied, we end up with a new and larger class of models. In particular, note that the diffusion terms in the state transition equations for $x_{2}(t)$ are allowed to be affine in the state variables. This makes the model different from and more general than- Vasicek type models even when $\bar{Q}=0$ and $D=0$. As our next result shows, the price of a zero-coupon bond can still be obtained in an explicit closed-form, and hence this interest rate model merits empirical verification along the lines of $[1],[3]$. By the risk-neutral pricing formula, the price of a zero-coupon bond $B(t, T)$ maturing at time $T$ is

$$
B(t, T)=\mathbb{E}\left[e^{-\int_{t}^{T} r(\tau) d \tau} \mid \mathcal{F}(t)\right]
$$

which after substituting the expression for the interest rate (2.17) becomes $B(t, T)=\mathbb{E}\left\{\exp \left[-\int_{t}^{T}\left[x_{1}^{\prime}(\tau) \bar{Q} x_{1}(\tau)+\bar{L}_{1}^{\prime} x_{1}(\tau)+\bar{L}_{2}^{\prime} x_{2}(\tau)\right] d \tau\right] \mid \mathcal{F}(t)\right\}$. 
However, this is the same as the cost functional (1.5) beginning at time $t$ (rather than zero), and with the following coefficients:

$$
\begin{aligned}
& \gamma=1, \quad S=0, \quad S_{1}=0, \quad S_{2}=0, \quad L_{u}=0, \quad X=0, \\
& Q=-2 \bar{Q}, \quad L_{1}=-2 \bar{L}_{1}, \quad L_{2}=-2 \bar{L}_{2}, \quad R=0 .
\end{aligned}
$$

The relevant differential equations from section 2.1 now become:

$$
\begin{gathered}
\left\{\begin{array}{l}
\dot{p}_{2}(t)+A_{2}^{\prime} p_{2}(t)-2 \bar{L}_{2}=0, \\
p_{2}(T)=0,
\end{array}\right. \\
\left\{\begin{array}{l}
\dot{P}(t)+A^{\prime} P(t)+P(t) A-2 \bar{Q}+\sum_{i=1}^{n_{2}} p_{2 i} Q_{i} \\
+\frac{1}{4} \sum_{j=1}^{n}\left[2 P(t) C_{j}+A_{3 j}^{\prime} p_{2}(t)\right]\left[2 C_{j}^{\prime} P(t)+p_{2}^{\prime}(t) A_{3 j}\right]=0, \\
P(T)=0,
\end{array}\right. \\
\left\{\begin{array}{l}
\dot{p}_{1}-2 \bar{L}_{1}+A^{\prime} p_{1}(t)+A_{1}^{\prime} p_{2}(t)+\frac{1}{2} \sum_{j=1}^{n}\left[2 P(t) C_{j}+A_{3 j}^{\prime} p_{2}(t)\right]\left[C_{j}^{\prime} p_{1}(t)+C_{1 j}^{\prime} p_{2}(t)\right]=0 \\
p_{1}(T)=0 \\
\dot{p}+\sum_{j=1}^{n} C_{j}^{\prime} P(t) C_{j}+\frac{1}{4} \sum_{j=1}^{n}\left[p_{1}^{\prime}(t) C_{j}+p_{2}^{\prime}(t) C_{1 j}\right]\left[C_{j}^{\prime} p_{1}(t)+C_{1 j}^{\prime} p_{2}(t)\right]=0 \\
p(T)=0
\end{array}\right.
\end{gathered}
$$

From Theorem 1 and its proof we immediately obtain the following result.

Corollary 1. Let the Riccati equation (2.19) have a unique solution for all $t \in[0, T]$. Under Assumptions 3-5 in sections 2.1-2.2, the price at time $t$ of a zero-coupon bond maturing at time $T$ is

$$
B(t, T)=\exp \left\{(1 / 2)\left[x_{1}(t)^{\prime} P(t) x_{1}(t)+p(t)+p_{1}^{\prime}(t) x_{1}(t)+p_{2}^{\prime}(t) x_{2}(t)\right]\right\},
$$

with $P(t), p(t), p_{1}(t)$ and $p_{2}(t)$ being solutions at time $t$ of the differential equations (2.19), (2.21), (2.20) and (2.18), respectively. 
This gives us a new and large class of interest rate models (which nests QATSM models in [2] and linear ATSM models such as those discussed in chapter 8 of [8]), for which we have a closed form bond pricing formula, as a by-product of our result on nonlinear risk-sensitive control. This raises two interesting problems: whether this is the largest class of QATSM type models and whether it brings any benefits, on real financial data, in terms of accurate yield curve modelling over existing models. Both these problems are topics of current research and are not explored here.

\section{Generalised risk-sensitive control}

\subsection{Problem formulation and solution}

In this section we consider a more general criterion. The generalisation consists in introducing noise dependent penalties on the control $u(t)$ and $x_{1}(t)$, as mentioned above. This kind of criterion was first introduced by the authors in [11], and it is a natural generalisation of the classical risk-sensitive cost functional. Hence consider the following generalised risk-sensitive criterion

$$
\begin{aligned}
\widetilde{J}(u(\cdot)) & =\gamma \mathbb{E}\left\{\operatorname { e x p } \left[\frac{\gamma}{2} x_{1}^{\prime}(T) S x_{1}(T)+\frac{\gamma}{2} \int_{0}^{T}\left[x_{1}^{\prime}(t) Q x_{1}(t)+u^{\prime}(t) R u(t)\right] d t\right.\right. \\
& +\frac{\gamma}{2} S_{1}^{\prime} x_{1}(T)+\frac{\gamma}{2} S_{2}^{\prime} x_{2}(T) \\
& +\frac{\gamma}{2} \int_{0}^{T}\left[L_{1}^{\prime} x_{1}(t)+L_{2}^{\prime} x_{2}(t)+L_{u}^{\prime} u(t)+u^{\prime}(t) X x_{1}(t)\right] d t \\
& \left.\left.+\frac{\gamma}{2} \int_{0}^{T}\left[x_{1}^{\prime}(t) Q_{x}+u^{\prime}(t) R_{u}\right] d W(t)\right]\right\}
\end{aligned}
$$

where we assume that

$$
Q_{x}(\cdot) \in L^{\infty}\left(0, T ; \mathbb{R}^{n_{1} \times n}\right), \quad R_{u}(\cdot) \in L^{\infty}\left(0, T ; \mathbb{R}^{m \times n}\right) .
$$

In this section we give the solution to the optimal control problem

$$
\left\{\begin{array}{l}
\min _{u(\cdot) \in \widetilde{\mathcal{A}}} \widetilde{J}(u(\cdot)), \\
\text { s.t. }(1.4) \text { holds, }
\end{array}\right.
$$


where, similarly to the previous section, we give the definition of the admissible set $\widetilde{\mathcal{A}}$ after introducing some necessary notation. We find the solution to (3.2) by the change of measure method, the main idea of which is to introduce a new probability measure, under which the original problem (3.2) can be transformed into a control problem the solution of which can be obtained by a direct application of Theorem 1 . Let us introduce the following notation for the rows of the corresponding matrices:

$$
Q_{x}^{\prime}=\left[\begin{array}{c}
q_{1} \\
\vdots \\
q_{n}
\end{array}\right], \quad R_{u}^{\prime}=\left[\begin{array}{c}
r_{1} \\
\vdots \\
r_{n}
\end{array}\right], \quad A_{3 j}=\left[\begin{array}{c}
a_{1 j} \\
\vdots \\
a_{n_{2 j}}
\end{array}\right], \quad B_{2 j}=\left[\begin{array}{c}
b_{1 j} \\
\vdots \\
b_{n_{2} j}
\end{array}\right]
$$

where $j=1, \ldots, n$. We define the stochastic process $\theta(t)$ as:

$$
\theta(t) \equiv-\frac{\gamma}{2}\left[Q_{x}^{\prime} x_{1}(t)+R_{u}^{\prime} u(t)\right]=\left[\begin{array}{c}
-\frac{\gamma}{2}\left(q_{1} x_{1}(t)+r_{1} u(t)\right) \\
\vdots \\
-\frac{\gamma}{2}\left(q_{n} x_{1}(t)+r_{n} u(t)\right)
\end{array}\right]=\left[\begin{array}{c}
\theta_{1} \\
\vdots \\
\theta_{n}
\end{array}\right]
$$

Define the process $Z^{u}(t)$ and the random variable $Z^{u}$ as:

$$
\begin{aligned}
Z^{u}(t) & \equiv \exp \left[-\int_{0}^{t} \theta^{\prime}(\tau) d W(\tau)-\frac{1}{2} \int_{0}^{t} \theta^{\prime}(\tau) \theta(\tau) d \tau\right], \\
Z^{u} & \equiv Z^{u}(T) .
\end{aligned}
$$

In order to ensure that $\mathbb{E}\left[Z^{u}\right]=1$, and thus $Z^{u}$ is a random variable that can be used to define an equivalent probability measure, we assume that the admissible controls satisfy the Novikov condition

C3. $\mathbb{E}\left[e^{\frac{1}{2} \int_{0}^{T} \theta^{\prime}(s) \theta(s) d s}\right]<\infty$.

We can now introduce an equivalent probability measure $\widetilde{\mathbb{P}^{u}}$ as

$$
\widetilde{\mathbb{P}^{u}}(\alpha) \equiv \int_{\alpha} Z^{u}(\omega) d \mathbb{P}(\omega), \quad \forall \alpha \in \mathcal{F}
$$

By Girsanov's theorem, the process

$$
\widetilde{W}^{u}(t) \equiv W(t)+\int_{0}^{t} \theta(\tau) d \tau,
$$


is a standard Brownian motion under the new probability measure $\widetilde{\mathbb{P}^{u}}$. Let $\widetilde{\mathbb{E}^{u}}$ denote the expectation under $\widetilde{\mathbb{P}^{u}}$. We can express criterion (3.1) in terms of this expectation as

$$
\begin{aligned}
\widetilde{J}(u(\cdot)) & =\widetilde{\mathbb{E}^{u}}\left\{\operatorname { e x p } \left[\frac{\gamma}{2} x_{1}^{\prime}(T) S x_{1}(T)+\frac{\gamma}{2} \int_{0}^{T}\left[x_{1}^{\prime}(t) Q x_{1}(t)+u^{\prime}(t) R u(t)\right] d t\right.\right. \\
& +\frac{\gamma}{2} S_{1}^{\prime} x_{1}(T)+\frac{\gamma}{2} S_{2}^{\prime} x_{2}(T) \\
& +\frac{\gamma}{2} \int_{0}^{T}\left[L_{1}^{\prime} x_{1}(t)+L_{2}^{\prime} x_{2}(t)+L_{u}^{\prime} u(t)+u^{\prime}(t) X x_{1}(t)\right] d t \\
& \left.\left.+\frac{\gamma}{2} \int_{0}^{T} \frac{\gamma}{4}\left(x_{1}^{\prime}(t) Q_{x}+u^{\prime}(t) R_{u}\right)\left(Q_{x}^{\prime} x_{1}(t)+R_{u}^{\prime} u(t)\right) d t\right]\right\}
\end{aligned}
$$

We now rearrange the terms of this criterion so that it has the form of (1.5). Note that

$$
\begin{aligned}
& x_{1}^{\prime}(t) Q x_{1}(t)+u^{\prime}(t) R u(t)+u^{\prime}(t) X x_{1}(t)+\frac{\gamma}{4}\left(x_{1}^{\prime}(t) Q_{x}+u^{\prime}(t) R_{u}\right)\left(Q_{x}^{\prime} x_{1}(t)+R_{u}^{\prime} u(t)\right) \\
= & x_{1}^{\prime}(t) Q x_{1}(t)+u^{\prime}(t) R u(t)+u^{\prime}(t) X x_{1}(t)+\frac{\gamma}{4}\left(x_{1}^{\prime}(t) Q_{x} Q_{x}^{\prime} x_{1}(t)+2 u^{\prime}(t) R_{u} Q_{x}^{\prime} x_{1}(t)\right. \\
+ & \left.u^{\prime}(t) R_{u} R_{u}^{\prime} u(t)\right)=x_{1}^{\prime}(t)\left(Q+\frac{\gamma}{4} Q_{x} Q_{x}^{\prime}\right) x_{1}(t) \\
+ & u^{\prime}(t)\left(X+\frac{\gamma}{2} R_{u} Q_{x}^{\prime}\right) x_{1}(t)+u^{\prime}(t)\left(R+\frac{\gamma}{4} R_{u} R_{u}^{\prime}\right) u(t) \\
= & x_{1}^{\prime}(t) \widetilde{Q} x_{1}(t)+u^{\prime}(t) \widetilde{X} x_{1}(t)+u^{\prime}(t) \widetilde{R} u(t),
\end{aligned}
$$

where

$$
\widetilde{Q}=Q+\frac{\gamma}{4} Q_{x} Q_{x}^{\prime}, \quad \widetilde{X}=X+\frac{\gamma}{2} R_{u} Q_{x}^{\prime}, \quad \widetilde{R}=R+\frac{\gamma}{4} R_{u} R_{u}^{\prime} .
$$

For later convenience, we also denote $\widetilde{L}_{1}=L_{1}, \widetilde{L}_{2}=L_{2}, \widetilde{L}_{u}=L_{u}, \widetilde{S}=S$, $\widetilde{S}_{1}=S_{1}, \widetilde{S}_{2}=S_{2}$. In what follows we will introduce further coefficients with a tilde overline, and for ease of referring, we will call the set of the coefficients without the tilde overline as $\mathcal{S}$, whereas the set of the coefficients with the tilde overline as $\widetilde{\mathcal{S}}$. 
The criterion (3.3) can now be written as:

$$
\begin{aligned}
\widetilde{J}(u(\cdot)) & =\gamma \widetilde{\mathbb{E}}\left\{\operatorname { e x p } \left[\frac{\gamma}{2} x_{1}^{\prime}(T) \widetilde{S} x_{1}(T)+\frac{\gamma}{2} \int_{0}^{T}\left[x_{1}^{\prime}(t) \widetilde{Q} x_{1}(t)+u^{\prime}(t) \widetilde{R} u(t)\right] d t\right.\right. \\
& +\frac{\gamma}{2} \widetilde{S}_{1}^{\prime} x_{1}(T)+\frac{\gamma}{2} \widetilde{S}_{2}^{\prime} x_{2}(T) \\
& \left.\left.+\frac{\gamma}{2} \int_{0}^{T}\left[\widetilde{L}_{1}^{\prime} x_{1}(t)+\widetilde{L}_{2}^{\prime} x_{2}(t)+\widetilde{L}_{u}^{\prime} u(t)+u^{\prime}(t) \widetilde{X} x_{1}(t)\right] d t\right]\right\}
\end{aligned}
$$

which is of the same form as (1.5) with the main difference being that the expectation is under $\widetilde{\mathbb{E}^{u}}$, and that the coefficients are those with a tilde overline. In order to make use of Theorem 1, we must also transform the system dynamics (1.4) so that it is expressed in terms of $\widetilde{W^{u}}(t)$. The equation for $x_{1}(t)$ can be written as:

$$
\begin{aligned}
d x_{1}(t) & =\left[A x_{1}(t)+B u(t)\right] d t+\sum_{j=1}^{n} C_{j} d W_{j}(t) \\
& =\left[A x_{1}(t)+B u(t)\right] d t+\sum_{j=1}^{n} C_{j}\left[\widetilde{d W^{u}}{ }_{j}(t)+\frac{\gamma}{2}\left(q_{j} x_{1}(t)+r_{j} u(t)\right) d t\right] \\
& =\left[\left(A+\frac{\gamma}{2} \sum_{j=1}^{n} C_{j} q_{j}\right) x_{1}(t)+\left(B+\frac{\gamma}{2} \sum_{j=1}^{n} C_{j} r_{j}\right) u(t)\right] d t \\
& +\sum_{j=1}^{n} C_{j} d \widetilde{W}_{j}(t) \\
& =\left(\widetilde{A} x_{1}(t)+\widetilde{B} u(t)\right) d t+\sum_{j=1}^{n} \widetilde{C}_{j} d \widetilde{W}_{j}(t),
\end{aligned}
$$

where

$$
\widetilde{A}=A+\frac{\gamma}{2} \sum_{j=1}^{n} C_{j} q_{j}, \quad \widetilde{B}=B+\frac{\gamma}{2} \sum_{j=1}^{n} C_{j} r_{j}, \quad \widetilde{C}_{j}=C_{j}, \quad j=1, \ldots, n .
$$


Writing the equation for $x_{2}(t)$ in terms of $\widetilde{W^{u}}(t)$ is more involved due to the nonlinearity. We begin by expressing the following vectors in more details:

$$
\begin{aligned}
& x_{1}^{\prime}(t) q_{j}^{\prime} A_{3 j} x_{1}(t)=\left[\begin{array}{c}
x_{1}^{\prime}(t) q_{j}^{\prime} a_{1 j} x_{1}(t) \\
\vdots \\
x_{1}^{\prime}(t) q_{j}^{\prime} a_{n_{2} j} x_{1}(t)
\end{array}\right]=\left[\begin{array}{c}
\frac{1}{2} x_{1}^{\prime}(t)\left(q_{j}^{\prime} a_{1 j}+a_{1 j}^{\prime} q_{j}\right) x_{1}(t) \\
\vdots \\
\frac{1}{2} x_{1}^{\prime}(t)\left(q_{j}^{\prime} a_{n_{2 j}}+a_{n_{2 j}}^{\prime} q_{j}\right) x_{1}(t)
\end{array}\right], \\
& u^{\prime}(t) r_{j}^{\prime} A_{3 j} x_{1}(t)=\left[\begin{array}{c}
u^{\prime}(t) r_{j}^{\prime} a_{1 j} x_{1}(t) \\
\vdots \\
u^{\prime}(t) r_{j}^{\prime} a_{n_{2} j} x_{1}(t)
\end{array}\right] \text {, } \\
& x_{1}^{\prime}(t) q_{j}^{\prime} B_{2 j} u(t)=\left[\begin{array}{c}
x_{1}^{\prime}(t) q_{j}^{\prime} b_{1 j} u(t) \\
\vdots \\
x_{1}^{\prime}(t) q_{j}^{\prime} b_{n_{2} j} u(t)
\end{array}\right] \text {, } \\
& u^{\prime}(t) r_{j}^{\prime} A_{3 j} x_{1}(t)+x_{1}^{\prime}(t) q_{j}^{\prime} B_{2 j} u(t)=\left[\begin{array}{c}
u^{\prime}(t)\left(r_{j}^{\prime} a_{1 j}+b_{1 j}^{\prime} q_{j}\right) x_{1}(t) \\
\vdots \\
u^{\prime}(t)\left(r_{j}^{\prime} a_{n_{2 j}}+b_{n_{2} j}^{\prime} q_{j}\right) x_{1}(t)
\end{array}\right] .
\end{aligned}
$$

We define $\widetilde{D}\left(x_{1}(t), u(t)\right)$ as:

$$
\begin{aligned}
\widetilde{D}\left(x_{1}(t), u(t)\right) \equiv & D\left(x_{1}(t), u(t)\right)+\frac{\gamma}{2} \sum_{j=1}^{n}\left[x_{1}^{\prime}(t) q_{j}^{\prime} A_{3 j} x_{1}(t)+u^{\prime}(t) r_{j}^{\prime} A_{3 j} x_{1}(t)+x_{1}^{\prime}(t) q_{j}^{\prime} B_{2 j} u(t)\right. \\
& \left.+u^{\prime}(t) r_{j}^{\prime} B_{2 j} u(t)\right]=\left[\begin{array}{c}
u^{\prime}(t) \widetilde{X}_{1} x_{1}(t)+u^{\prime}(t) \widetilde{R}_{1} u(t)+x_{1}^{\prime}(t) \widetilde{Q}_{1} x_{1}(t) \\
\vdots \\
u^{\prime}(t) \widetilde{X}_{n_{2}} x_{1}(t)+u^{\prime}(t) \widetilde{R}_{n_{2}} u(t)+x_{1}^{\prime}(t) \widetilde{Q}_{n_{2}} x_{1}(t)
\end{array}\right],
\end{aligned}
$$

where for $i=1, \ldots, n_{2}$ :

$$
\begin{aligned}
& \widetilde{X}_{i}=X_{i}+\frac{\gamma}{2} \sum_{j=1}^{n}\left(r_{j}^{\prime} a_{i j}+b_{i j}^{\prime} q_{j}\right) \\
& \widetilde{R}_{i}=R_{i}+\frac{\gamma}{4} \sum_{j=1}^{n}\left(r_{j}^{\prime} b_{i j}+b_{i j}^{\prime} r_{j}\right) \\
& \widetilde{Q}_{i}=Q_{i}+\frac{\gamma}{4} \sum_{j=1}^{n}\left(q_{j}^{\prime} a_{i j}+a_{i j}^{\prime} q_{j}\right) .
\end{aligned}
$$


The equation for $x_{2}(t)$ can now be written as:

$$
\begin{aligned}
d x_{2}(t) & =\left[A_{1} x_{1}(t)+A_{2} x_{2}(t)+D\left(x_{1}(t), u(t)\right)+B_{1} u(t)\right] d t \\
& +\sum_{i=1}^{n}\left[A_{3 j} x_{1}(t)+B_{2 j} u(t)+C_{1 j}\right]\left[\widetilde{d W^{u}}{ }_{j}(t)+\frac{\gamma}{2}\left(q_{j} x_{1}(t)+r_{j} u(t)\right) d t\right] \\
& =\left[\left(A_{1}+\frac{\gamma}{2} \sum_{j=1}^{n} C_{1 j} q_{j}\right) x_{1}(t)+A_{2} x_{2}(t)+\widetilde{D}\left(x_{1}(t), u(t)\right)\right. \\
& \left.+\left(B_{1}+\frac{\gamma}{2} \sum_{j=1}^{n} C_{1 j} r_{j}\right) u(t)\right] d t+\sum_{j=1}^{n}\left(A_{3 j} x_{1}(t)+B_{2 j} u(t)+C_{1 j}\right) d \widetilde{W}_{j}(t) \\
& =\left[\widetilde{A}_{1}+\widetilde{A}_{2} x_{2} t+\widetilde{D}\left(x_{1}(t), u(t)\right)+\widetilde{B}_{1} u(t)\right] d t \\
& +\sum_{j=1}^{n}\left[\widetilde{A}_{3 j} x_{1}(t)+\widetilde{B}_{2 j} u(t)+\widetilde{C}_{1 j}\right] d \widetilde{W}_{j}^{u}(t),
\end{aligned}
$$

where

$$
\begin{array}{ll}
\widetilde{A}_{1}=A_{1}+\frac{\gamma}{2} \sum_{j=1}^{n} C_{1 j} q_{j}, & \widetilde{B}_{1}=B_{1}+\frac{\gamma}{2} \sum_{j=1}^{n} C_{1 j} r_{j}, \\
\widetilde{A}_{2}=A_{2}, \quad \widetilde{A}_{3 j}=A_{3 j}, & \widetilde{B}_{2 j}=B_{2 j}, \quad \widetilde{C}_{1 j}=C_{1 j},
\end{array}
$$

with $j=1, \ldots, n$. The conditions $\mathrm{C} 1(\mathrm{q})$ and $\mathrm{C} 2(\mathrm{q})$ for the coefficients of the set $\widetilde{\mathcal{S}}$ we denote by $\widetilde{C}_{1}(q)$ and $\widetilde{C}_{2}(q)$, respectively, and make the following assumption.

Assumption 6. Assumptions 1 and 2 hold for the set $\widetilde{\mathcal{S}}$.

We can now define the set of admissible control processes $\widetilde{\mathcal{A}}$.

Definition 2. Let

$$
\widetilde{\mathcal{A}}_{q}=\left\{u(\cdot) \in L_{\mathcal{F}}^{2}\left(0, T ; \mathbb{R}^{m}\right): C 3, \widetilde{C}_{1}(q) \text {, and } \widetilde{C}_{2}(q) \text { hold }\right\} .
$$

The set of admissible controls $\widetilde{\mathcal{A}}$ for the problem (3.2) is

$$
\widetilde{\mathcal{A}}=\bigcup_{q>1} \widetilde{\mathcal{A}}_{q}
$$


Note that the problem of minimizing (3.4) subject to (3.5), (3.6), which is equivalent to (3.2), is similar to the problem (2.1) with the coefficients from the set $\widetilde{\mathcal{S}}$. The only difference is that in (3.4) the probability measure $\widetilde{\mathbb{P}^{u}}$ depends on the control. However, for each control satisfying C3, and the corresponding $\widetilde{\mathbb{P}^{u}}$, the process $\widetilde{W^{u}}(t)$ is a Brownian motion, and similarly for problem (2.1), i.e. for each control process $u(t)$, and the probability measure $\mathbb{P}$, the process $W(t)$ is a Brownian motion. Therefore, the two problems have the same solution. This means that we can use Theorem 1 to find the solution to problem of minimizing (3.4) subject to (3.5), (3.6). First we need to derive some conditions that ensure a control process (2.7) satisfies condition $\mathrm{C} 3$. We introduce the coefficients:

$$
\begin{aligned}
K_{0 \theta} & =-\frac{\gamma}{2} R_{u}^{\prime} K_{0}, \quad K_{1 \theta}=-\frac{\gamma}{2}\left(Q_{x}^{\prime}+R_{u}^{\prime} K_{1}\right), \\
\widetilde{M}_{1} & =\left[2 \widetilde{M} C_{1}, \ldots, 2 \widetilde{M} C_{n}\right], \quad \widetilde{N}_{1}=\left[2 \widetilde{N}^{\prime} C_{1}, \ldots, 2 \widetilde{N}^{\prime} C_{n}\right],
\end{aligned}
$$

where $\widetilde{M}$ and $\widetilde{N}$ are solutions to the following Riccati and linear differential equations, respectively:

$$
\begin{aligned}
& \left\{\begin{array}{l}
\dot{\widetilde{M}}+\widetilde{M} \bar{A}+\bar{A}^{\prime} \widetilde{M}+\frac{1}{2} K_{1 \theta}^{\prime} K_{1 \theta}+\frac{1}{2} \widetilde{M}_{1} \widetilde{M}_{1}^{\prime}=0 \\
\widetilde{M}(T)=0
\end{array}\right. \\
& \left\{\begin{array}{l}
\dot{\widetilde{N}}+\bar{A}^{\prime} \widetilde{N}+\widetilde{M}_{1} \widetilde{N}_{1}^{\prime}+K_{1 \theta}^{\prime} K_{0 \theta}+2 \widetilde{M} \bar{B}=0 \\
\widetilde{N}(T)=0 .
\end{array}\right.
\end{aligned}
$$

Assumption 7. There exists a unique solution to (3.7) such that $P_{0}^{-1}>$ $2 \widetilde{M}(0)$.

Lemma 2. If Assumption 7 holds, then $\bar{u}(t)$ satisfies $C 3$. 
Proof. Let $u(t)=\bar{u}(t)$. Then we have:

$$
\begin{aligned}
& \mathbb{E}\left[e^{\frac{1}{2} \int_{0}^{T} \theta^{\prime}(s) \theta(s) d s}\right]=\mathbb{E}\left[e^{\left.x_{1}^{\prime}(0) \widetilde{M}(0) x_{1}(0)+x_{1}^{\prime}(0) \widetilde{N}(0)+\int_{0}^{T}\left[\sum_{j=1}^{n}\left(C_{j}^{\prime} \widetilde{M} C_{j}\right)+\widetilde{N}^{\prime} \bar{B}+\frac{1}{2} \widetilde{N}_{1} \widetilde{N}_{1}^{\prime}\right] d t\right]}\right. \\
& \times \mathbb{E}\left[e^{\left.\int_{0}^{T}\left(x_{1}^{\prime} \widetilde{M}_{1}+\widetilde{N}_{1}\right) d W-\frac{1}{2} \int_{0}^{T}\left(x_{1}^{\prime} \widetilde{M}_{1}+\widetilde{N}_{1}\right)\left(\widetilde{M}_{1}^{\prime} x_{1}+\widetilde{N}_{1}^{\prime}\right) d t\right]}\right. \\
& \leq e^{\int_{0}^{T}\left[\sum_{j=1}^{n}\left(C_{j}^{\prime} \widetilde{M} C_{j}\right)+\widetilde{N}^{\prime} \bar{B}+\frac{1}{2} \widetilde{N}_{1} \widetilde{N}_{1}^{\prime}\right] d t} \mathbb{E}\left[e^{x_{1}^{\prime}(0) \widetilde{M}(0) x_{1}(0)+x_{1}^{\prime}(0) \widetilde{N}(0)}\right] \\
& =e^{\int_{0}^{T}\left[\sum_{j=1}^{n}\left(C_{j}^{\prime} \widetilde{M} C_{j}\right)+\widetilde{N}^{\prime} \bar{B}+\frac{1}{2} \widetilde{N}_{1} \widetilde{N}_{1}^{\prime}\right] d t} \\
& \times \int_{\mathbb{R}^{n_{1}}} e^{x^{\prime} \widetilde{M}(0) x+x^{\prime} \tilde{N}(0)} \frac{1}{(2 \pi)^{n_{1} / 2}\left|P_{0}\right|^{1 / 2}} e^{-\frac{1}{2}\left(x-\mu_{0}\right)^{\prime} P_{0}^{-1}\left(x-\mu_{0}\right)} d x \\
& =\frac{e^{-\frac{1}{2} \mu_{0}^{\prime} P_{0}^{-1} \mu_{0}-\frac{1}{2}\left(\widetilde{N}(0)+P_{0}^{-1} \mu_{0}\right)^{\prime}\left(P_{0}-2 \widetilde{M}(0)\right)^{-1}\left(\widetilde{N}(0)+P_{0}^{-1} \mu_{0}\right)+\int_{0}^{T}\left[\sum_{j=1}^{n}\left(C_{j}^{\prime} \widetilde{M} C_{j}\right)+\widetilde{N}^{\prime} \bar{B}+\frac{1}{2} \widetilde{N}_{1} \widetilde{N}_{1}^{\prime}\right] d t}}{\left|\left(P_{0}^{-1}-2 \widetilde{M}(0)\right)\right|^{1 / 2}\left|P_{0}\right|^{1 / 2}} \\
& \times \int_{\mathbb{R}^{n_{1}}} \frac{e^{-\frac{1}{2}\left[x-\left(P_{0}^{-1}-2 \widetilde{M}(0)\right)^{-1}\left(\widetilde{N}(0)+P_{0}^{-1} \mu_{0}\right)\right]^{\prime}\left(P_{0}^{-1}-2 \widetilde{M}(0)\right)\left[x-\left(P_{0}^{-1}-2 \widetilde{M}(0)\right)^{-1}\left(\widetilde{N}(0)+P_{0}^{-1} \mu_{0}\right)\right]}}{(2 \pi)^{n_{1} / 2}\left|\left(P_{0}^{-1}-2 \widetilde{M}(0)\right)\right|^{-1 / 2}} d x \\
& =\frac{e^{-\frac{1}{2} \mu_{0}^{\prime} P_{0}^{-1} \mu_{0}-\frac{1}{2}\left(\widetilde{N}(0)+P_{0}^{-1} \mu_{0}\right)^{\prime}\left(P_{0}-2 \widetilde{M}(0)\right)^{-1}\left(\widetilde{N}(0)+P_{0}^{-1} \mu_{0}\right)+\int_{0}^{T}\left[\sum_{j=1}^{n}\left(C_{j}^{\prime} \widetilde{M} C_{j}\right)+\widetilde{N}^{\prime} \bar{B}+\frac{1}{2} \widetilde{N}_{1} \widetilde{N}_{1}^{\prime}\right] d t}}{\left|\left(P_{0}^{-1}-2 \widetilde{M}(0)\right)\right|^{1 / 2}\left|P_{0}\right|^{1 / 2}},
\end{aligned}
$$

where the first equality above is obtained using similar steps to the section (2.2), and thus the detailed derivation is omitted.

We can now state the solution to problem (3.2) which obtained by a direct application of Theorem 1 .

Theorem 2. Let the Assumption 6 hold, and let $\widetilde{\bar{R}}, \widetilde{\bar{Y}}, \widetilde{\bar{X}}$, denote the coefficients $\bar{R}, \bar{Y}, \bar{X}$, corresponding to the set $\widetilde{\mathcal{S}}$, respectively. For the choice of the coefficients $K_{0}(t)$ and $K_{1}(t)$ as

$$
K_{0}=-\frac{1}{2} \widetilde{\bar{R}}^{-1} \widetilde{\widetilde{Y}}, \quad K_{1}=-\frac{1}{2} \widetilde{\bar{R}}^{-1} \widetilde{\bar{X}} x_{1}(t),
$$

let the Assumption 3-5 and Assumption 7 hold for the coefficients of the set $\widetilde{\mathcal{S}}$. Then there exists a unique solution to problem (3.2) given by

$$
\widetilde{u}^{*}(t)=K_{0}+K_{1} x_{1}(t) .
$$

Remark 3. If the process $x_{1}(t)$ is deterministic under the control $\widetilde{u}^{*}(t)$, then it is not necessary to have Assumptions 3-5 and Assumption 7 for the conclusions of Theorem 2 to hold, since it is clear from Remark 2 and condition C3 that $\widetilde{u}^{*}(t)$ is admissible in this case. 


\subsection{Application to optimal investment}

As already indicated in the introduction, a special case of the risk-sensitive control problem (2.1) is that of exponential utility maximization. In this section we propose a natural generalisation of the expected utility as a criterion for optimal investment. The resulting problem is an example of the generalised risk-sensitive control problem (3.2), and thus Theorem 2 is used to obtain the solution.

Consider a market model consisting of a risk-free asset $S_{0}(t)$ and the risky assets $S_{i}(t), i=1,2, \ldots, m$. These assets are the solutions to the following equations:

$$
\left\{\begin{array}{l}
d S_{0}(t)=S_{0}(t) r d t \\
d S_{i}(t)=S_{i}(t)\left[\mu_{i} d t+\sum_{j=1}^{n} \sigma_{i j} d W_{j}(t)\right], \quad i=1, \ldots, m \\
S_{i}(0)>0 \text { is given for all } i=0, \ldots, m
\end{array}\right.
$$

where we assume that

$$
r(\cdot), \mu_{i}(\cdot), \sigma_{i j}(\cdot) \in L^{\infty}(0, T ; \mathbb{R}), \quad i=1, \ldots, m, \quad j=1, \ldots, n .
$$

We pack the volatility coefficients $\sigma_{i j}$ in the following more convenient form:

$$
\begin{aligned}
\sigma_{i} & \equiv\left[\sigma_{1 i}, \sigma_{2 i}, \ldots, \sigma_{m i}\right], \quad i=1, \ldots, n, \\
\sigma & \equiv\left[\sigma_{1}^{\prime}, \sigma_{2}^{\prime}, \ldots, \sigma_{n}^{\prime}\right] .
\end{aligned}
$$

The equation describing the value $y(t)$ of a self-financing portfolio in such a market is

$$
\begin{aligned}
d y(t) & =[r y(t)+b u(t)] d t+\sum_{j=1}^{n} \sigma_{j} u(t) d W_{j}(t) \\
& =[r y(t)+b u(t)] d t+u^{\prime}(t) \sigma d W(t),
\end{aligned}
$$

where $y(0)=y_{0}$, the investors initial wealth, is given; $b \equiv\left[\mu_{1}-r, \ldots, \mu_{m}-r\right]$; and $u_{i}(t)$ is the amount of wealth invested in asset $i$. The optimal investment 
problem with exponential utility is defined as:

$$
\left\{\begin{array}{l}
\min _{u(\cdot) \in L_{\mathcal{F}}^{2}\left(0, T ; \mathbb{R}^{m}\right)} \mathbb{E}\left[e^{-a y(T)}\right], \\
\text { s.t. } \quad(3.9) \text { holds, }
\end{array}\right.
$$

where $0<a \in \mathbb{R}$ is some given coefficient. The solution to this problem is well-known and was first obtained by Merton in [29], [30].

The integral form of equation (3.10) is

$$
y(T)=y_{0}+\int_{0}^{T}[r y(\tau)+b u(\tau)] d \tau+\int_{0}^{T} u^{\prime}(\tau) \sigma d W(\tau) .
$$

Substituting this into the criterion of problem (3.11) we get

$$
\begin{aligned}
& \mathbb{E}\left[\exp \left(-a y_{0}-\int_{0}^{T}[\operatorname{ary}(\tau)+a b u(\tau)] d \tau-\int_{0}^{T} u^{\prime}(\tau) a \sigma d W(\tau)\right)\right] \\
\sim & \mathbb{E}\left[\exp \left(\int_{0}^{T}[(-a r) y(\tau)+a b u(\tau)] d \tau+\int_{0}^{T} u^{\prime}(\tau)(-a \sigma) d W(\tau)\right)\right],
\end{aligned}
$$

where $\sim$ indicated that both the criteria give the same optimal control. Clearly, criterion (3.12) is just an example of the generalised risk-sensitive criterion (3.1). The parameter $a$, which is chosen by the investor, can adjust the coefficients $a r(t), a b(t), a \sigma(t)$, but obviously not in an arbitrary manner. In order to give the investor more flexibility in choosing the coefficients of the optimal investment criterion, we propose the following natural generalisation of (3.12):

$$
\widehat{J}(u(\cdot)) \equiv \mathbb{E}\left[\exp \left(\int_{0}^{T}\left[\widehat{L} y(\tau)+\widehat{L}_{u}^{\prime} u(\tau)\right] d \tau+\int_{0}^{T} u^{\prime}(\tau) \widehat{R} d W(\tau)\right)\right],
$$

where the coefficients are assumed to belong to the following spaces:

$$
\widehat{L}(\cdot) \in L^{\infty}(0, T ; \mathbb{R}), \quad \widehat{L}_{u}(\cdot) \in L^{\infty}\left(0, T ; \mathbb{R}^{m}\right), \quad \widehat{R}(\cdot) \in L^{\infty}\left(0, T ; \mathbb{R}^{m \times n}\right) .
$$

The optimal investment problem for this criterion is:

$$
\left\{\begin{array}{l}
\min _{u(\cdot) \in L_{\mathcal{F}}^{2}\left(0, T ; \mathbb{R}^{m}\right)} \widehat{J}(u(\cdot)) \\
\text { s.t. } \quad(3.9) \text { holds }
\end{array} .\right.
$$


However, this is just an important example of the control problem (3.2) with $x_{2}(t)=y(t)$ and the following coefficients:

$$
\begin{aligned}
& n_{2}=1, \quad A=0, \quad B=0, \quad C_{j}=0, \quad A_{1}=0, \quad A_{2}=r, \quad, Q_{1}=0, \quad X_{1}=0, \\
& B_{1}=b, \quad A_{3 j}=0, \quad B_{2 j}=\sigma_{j}, \quad C_{1 j}=0, \quad \gamma=1, \quad S=0, \quad S_{1}=0, \quad S_{2}=0, \\
& R=0, \quad L_{1}=0, \quad L_{2}=2 \widehat{L}, \quad L_{u}=2 \widehat{L}_{u}, \quad Q_{x}=0, \quad R_{u}=2 \widehat{R} \equiv\left[\widehat{r}_{1}^{\prime}, \ldots, \widehat{r}_{n}^{\prime}\right], \\
& X=0, \quad R_{1}=0, \quad Q=0 .
\end{aligned}
$$

From these, we obtain the coefficients of the set $\widetilde{\mathcal{S}}$ as:

$$
\begin{aligned}
& \widetilde{Q}=0, \quad \widetilde{X}=0, \quad \widetilde{R}=\widehat{R} \widehat{R}^{\prime}, \quad \widetilde{L}_{1}=0, \quad \widetilde{L}_{2}=2 \widehat{L}, \quad \widetilde{L}_{u}=2 \widehat{L}_{u}, \quad \widetilde{S}=0, \quad \widetilde{S}_{1}=0, \\
& \widetilde{A}=0, \quad \widetilde{B}=0, \quad \widetilde{C}=0, \quad \widetilde{Q}_{1}=0, \quad \widetilde{X}_{1}=0, \quad \widetilde{R}_{1}=\frac{1}{2} \sum_{j=1}^{n} \sigma_{j} \widehat{r}_{j}, \quad \widetilde{A}_{1}=0, \quad \widetilde{B}_{1}=b, \\
& \widetilde{A}_{3 j}=0, \quad \widetilde{B}_{2 j}=\sigma_{j}, \quad \widetilde{C}_{1 j}=0, \quad \widetilde{C}_{j}=0, \quad \widetilde{A}_{2}=r, \quad \widetilde{S}_{2}=0 .
\end{aligned}
$$

Equation (2.2) now becomes:

$$
\left\{\begin{array}{l}
\dot{p}_{2}(t)+r p_{2}(t)+2 \widehat{L}=0, \\
p_{2}(T)=0
\end{array}\right.
$$

whereas the coefficients $\widetilde{\bar{R}}, \widetilde{\bar{X}}, \widetilde{\bar{Y}}, \widetilde{\bar{Z}}$, are

$$
\begin{aligned}
& \widetilde{\bar{R}}=\widehat{R} \widehat{R}^{\prime}+\frac{p_{2}(t)}{2} \sum_{j=1}^{n} \sigma_{j} \widehat{r}_{j}^{\prime}+\frac{p_{2}^{2}(t)}{4} \sum_{j=1}^{n} \sigma_{j}^{\prime} \sigma_{j}, \\
& \widetilde{\bar{X}}=0, \quad \widetilde{\bar{Y}}=2 \widehat{L}_{u}+p_{2}(t) b^{\prime}, \quad \widetilde{\bar{Z}}=0 .
\end{aligned}
$$

The Assumption 1 now becomes:

Assumption 8. $\widetilde{\bar{R}}(t)>0$, a.e. $t \in[0, T]$. 
It is easy to show that equation (2.4) has a unique solution equal to zero in this case. Hence Assumption 6 holds. Is is also true that (2.6), (2.5) have a unique solution equal to zero. Since $x_{1}(t)=0 \forall t \in[0, T]$, the optimal investment strategy $\widetilde{u}^{*}(t)$ follows directly from Theorem 2 and Remark 3 .

Corollary 2. Let the Assumption 3 hold. There exists a unique solution to the optimal investment problem (3.14) given by

$$
\widetilde{u}^{*}(t)=-\frac{1}{2}\left[\widehat{R} \widehat{R}^{\prime}+\frac{p_{2}(t)}{2} \sum_{j=1}^{n} \sigma_{j} \widehat{r}_{j}^{\prime}+\frac{p_{2}^{2}(t)}{4} \sum_{j=1}^{n} \sigma_{j}^{\prime} \sigma_{j}\right]^{-1}\left[2 \widehat{L}_{u}+p_{2}(t) b^{\prime}\right] .
$$

We do not explore the possible economic interpretation of this generalisation here, since our motivation is simply to see how far we can go while still obtained simple closed-form solutions of increasingly general class of systems and cost functions.

\section{Conclusions}

In this paper we consider the risk-sensitive control problem for a class of nonlinear systems with multiplicative noise. Under certain reasonable assumptions, the complete solution to such an optimal control problem is obtained in an explicit closed-form. The generalised version of the criterion that includes noise dependent penalties on the control and state is also solved in an explicit closed form by using a change of measure approach. We propose two different possible applications of these results. First, we propose a new interest rate model and derive the price of a zero-coupon bond in an explicit closed-form. Second, we propose a generalisation of the classical optimal investment problem with exponential utility. Both these applications are worthy of further exploration, in terms of economic interpretation as well as empirical studies. One further possibility for application to optimal investment would be to include the state $x_{1}(t)$ as a model for the image of the product, similarly to [18], [28]. In this case the aim of the company that produces the new product and invests in a market, would be to maximise its exponential utility and ensure that the image of its product changes according to a desired policy. This application will be considered in a future paper. 


\section{Acknowledgement}

The authors are grateful to the reviewer for a careful reading of their

article, and for several very useful remarks which have lead to a significantly improved version of the paper.

\section{References}

[1] Ahn, D.-H., and Gao, B., A parametric nonlinear model of term structure dynamics, The Review of Financial Studies, 12, No. 4, 721-762 (1999)

[2] Ahn, D.-H., Dittmar, R. F., and Gallant, A. R., Quadratic term structure models: theory and evidence, The Review of Financial Studies, 15, No. 1, 243-288 (2002).

[3] Ait-Sahalia, Y., Testing continuous-time models of the spot interest rate, The Review of Financial Studies, 9, No. 2, 385-426 (1996).

[4] Anderson, B. D. O., and Moore, J., Optimal control: linear quadratic methods, Prentice Hall, (1990).

[5] Basu, A., Bhattacharyya, T., and Borkar, V. S., A learning algorithm for risk-sensitive cost, Mathematics of Operations Research, 33, No. 4, 880-898 (2008).

[6] Bensoussan, A., and Van Schuppen, J. H., Optimal control of partially observable stochastic systems with an exponential-of-integral performance index, SIAM J. Control and Optimisation, 23, 599-613 (1985).

[7] Bielecki, T. R., Pliska, S. R., and Sheu, S. J., Risk sensitive portfolio management with Cox-Ingersoll-Ross interest rates: the HJB equation, SIAM J. Control and Optimization, 44, 1811-1843 (2005).

[8] Bingham, N., and Kiesel, R., Risk-neutral Valuation, Springer Finance, Second Edition, 2004.

[9] Borkar, V. S., and Meyn, S. P., Risk-sensitive optimal control for Markov decision processes with monotone cost, Mathematics of Operations Research, 27, No. 1, 192-209 (2002). 
[10] Cavazos-Cadena, R., and Hernández-Hernández, D., Discounted approximations for risk-sensitive average criteria in Markov decision chains with finite state space, Mathematics of Operations Research, 36, No. 1, 133146 (2011).

[11] Date, P., and Gashi, B., Generalised risk-sensitive control with full and partial state observation, Journal of Mathematical Modelling and Algorithms, to appear.

[12] Davis, M. H. A., and Lleo, S., Jump-diffusion risk-sensitive asset management I: diffusion factor model, SIAM J. Fin. Math., 2, 22-54 (2011).

[13] Duncan, T.E., and Pasik-Duncan, B., Linear-exponential-quadratic Gaussian control for stochastic equations in Hilbert space, Dynamic Systems and Applications, 21, 407-416 (2012).

[14] Glover, K., anf J. Doyle, State space formulae for all stabilizing controllers that satisfy an $H_{\infty}$ norm bound and relation to risk-sensitivity, Systems $\&$ Control Letters, 11, 167-172, (1988).

[15] Glover, K., Minimum entropy and risk-sensitive control: the continuous time case, Proceedings of the 28th IEEE Conference on Decision and Control, 388-391, 1989.

[16] Goel, M., and Kumar, K. S., Risk-sensitive portfolio optimization problems with fixed income securities, Journal of Optimization Theory and Applications, 142, 1, 67-84 (2009).

[17] Huang, J., Li, X., and Wang, G., Maximum principles for a class of partial information risk-sensitive optimal controls, IEEE Trans. Automatic Control, 55, 6, 1438-1443, (2010).

[18] Jack, A., Jonhnson, T. C., and Zervos, M., A Singular Control Problem with Application to the Goodwill Problem,Stochastic Processes and their Applications, 118, 2098-2124, (2008).

[19] Jacobson, D. H., Optimal stochastic linear systems with exponential criteria and their relation to deterministic differential games, IEEE Trans. Automatic Control, AC-18, 124-131 (1973). 
[20] Jingtao, S, anf Zhen, W., A risk sensitive stochastic maximum principle for optimal control of jump diffusions and its applications, Acta Mathematica Scientia, 31B(2), 419-433 (2011).

[21] Kloeden, P. E., and Platen, E., Numerical solution of stochastic differential equations, Springer-Verlag, Berlin, 1992.

[22] Kraniak, J. C., Machell, F. M., Markus, S. I., and Spreyer, J. L., The dynamic linear expoential gaussian team problem, IEEE Trans. Automatic Control, 27, 860-869, (1982).

[23] Kumar, P. R., and van Schuppen, J. H., On the optimal control of stochastic systems with an exponential-of-integral performance index, J. Math. Anal. Appl., 80, 312-332, (1981).

[24] Kuroda, K., and Nagai, H., Risk-sensitive portfolio optimization on infinite time horizon, Stochastics and Stochastic Reports, 73, 309-331 (2002).

[25] Levendorskii, S., Pseudodiffusions and quadratic term structure models, Mathematical Finance, 15, 393-424 (2005).

[26] Lim, A. E. B., Zhou, X. Y., and Moore, J. B., Multiple-objective risk-sensitive control and its small noise limit, Automatica, 39, 533-541 (2003).

[27] Lim, A. E. B., and Zhou, X. Y., A new risk-sensitive maximum principle, IEEE Trans. Automatic Control, 50, 7, 958-966 (2005).

[28] Lon, P. C., and Zervos, M., A model for optimally advertising and launching a product, Mathematics of Operations Research, 36, 363-376 (2011)

[29] Merton, R. C., Analytical optimal control theory as applied to stochastic and non-stochastic economics, Ph. D. Thesis, MIT, 1970.

[30] Merton, R. C., Optimum consumption and portfolio rules in a continuous time model, J. Economic Theory, 3, 373-413, (1971). 
[31] Speyer, J. L., Deyst, J., and Jacobson, D. H., Optimization of stochastic linear systems with additive measurement and process noise using expoential performance criteria, IEEE Trans. Automatic Control, AC-19, 358-366, (1974).

[32] Speyer, J. L., An adaptive terminal guidance scheme based on an exponential cost criterion with application to homing missile gidance, IEEE Trans. Automatic Control, AC-21, 371-375, (1976).

[33] Whittle, P, Risk-sensitive linear/quadratic/Gaussian control, Advances in Applied Probability, 13, 764-777 (1981).

[34] Whittle, P., Risk-sensitive optimal control, Wiley, (1990).

[35] Wonham, W. M., Random differential equations in control theory, In $A$. T. Bharucha-Reid Ed., Probabilistic methods in applied mathematics, Vol. 2, 132-212, Academic Press, New York, 1970.

[36] Yong, J. and Zhou, X. Y., Stochastic controls: Hamiltonian systems and HJB equations, Springer, 1999. 